\title{
Self-consistent two-phase AGN torus models ${ }^{\star} \star \star$ SED library for observers
}

\author{
Ralf Siebenmorgen ${ }^{1}$, Frank Heymann ${ }^{2}$, and Andreas Efstathiou ${ }^{3}$ \\ ${ }^{1}$ European Southern Observatory, Karl-Schwarzschild-Str. 2, 85748 Garching b. München, Germany \\ e-mail: Ralf.Siebenmorgen@eso.org \\ 2 Deutsches Zentrum für Luft- und Raumfahrt, Kalkhorstweg 53, 17235 Neustrelitz, Germany \\ 3 School of Sciences, European University Cyprus, Diogenes Street, Engomi, 1516 Nicosia, Cyprus
}

Received 6 March 2015 / Accepted 20 August 2015

ABSTRACT

\begin{abstract}
We assume that dust near active galactic nuclei (AGNs) is distributed in a torus-like geometry, which can be described as a clumpy medium or a homogeneous disk, or as a combination of the two (i.e. a two-phase medium). The dust particles considered are fluffy and have higher submillimeter emissivities than grains in the diffuse interstellar medium. The dust-photon interaction is treated in a fully self-consistent three-dimensional radiative transfer code. We provide an AGN library of spectral energy distributions (SEDs). Its purpose is to quickly obtain estimates of the basic parameters of the AGNs, such as the intrinsic luminosity of the central source, the viewing angle, the inner radius, the volume filling factor and optical depth of the clouds, and the optical depth of the disk midplane, and to predict the flux at yet unobserved wavelengths. The procedure is simple and consists of finding an element in the library that matches the observations. We discuss the general properties of the models and in particular the $10 \mu \mathrm{m}$ silicate band. The AGN library accounts well for the observed scatter of the feature strengths and wavelengths of the peak emission. AGN extinction curves are discussed and we find that there is no direct one-to-one link between the observed extinction and the wavelength dependence of the dust cross sections. We show that objects in the library cover the observed range of mid-infrared colors of known AGNs. The validity of the approach is demonstrated by matching the SEDs of a number of representative objects: Four Seyferts and two quasars for which we present new Herschel photometry, two radio galaxies, and one hyperluminous infrared galaxy. Strikingly, for the five luminous objects we find that pure AGN models fit the SED without needing to postulate starburst activity.
\end{abstract}

Key words. quasars: general - radiative transfer - infrared: galaxies - galaxies: Seyfert - methods: numerical

\section{Introduction}

The suggestion that the $2.2-22 \mu \mathrm{m}$ emission of Seyfert galaxies and quasars may be due to dust (Low \& Kleinmann 1968) was first explored analytically by Rees et al. (1969) who were able to reproduce the slope of the near- to mid-infrared (near-IR, mid-IR) spectrum. Radiative transfer models of spherically symmetric distributions of dust in AGNs were first developed by Rowan-Robinson \& Crawford (1989), Lawrence et al. (1991), and Rowan-Robinson \& Efstathiou (1993).

Trying to account for the infrared (IR) emission of type 1 and type 2 AGNs with models that assume a disk-like or toroidal distribution of dust was recognized early on as an important test of the unified model for AGNs (Antonucci 1993). These models have been developed since the early 1990s (Efstathiou \& Rowan-Robinson 1991, 1995; Pier \& Krolik 1992, 1993; Rowan-Robinson et al. 1993; Granato \& Danese 1994; Efstathiou et al. 1995). Most of the discussion on these models concerned the interpretation of the observed behavior of the silicate feature at $9.7 \mu \mathrm{m}$ that was absent in emission in type 1

\footnotetext{
* Herschel is an ESA space observatory with science instruments provided by European-led Principal Investigator consortia and with important participation from NASA.

$\star \star$ The SED library of the AGN models is available at the CDS via anonymous ftp to cdsarc.u-strasbg.fr (130.79.128.5) or via http://cdsarc.u-strasbg.fr/viz-bin/qcat?]/A+A/583/A120, and at http://www.eso.org/ rsiebenm/agn_models/
}

objects (Roche et al. 1991) and challenged the whole idea of trying to explain the IR emission of AGNs with dust models and the unified model itself. It is now recognized that smooth and clumpy torus models (Feltre et al. 2012) can reproduce the whole range of features that are observed using Spitzer spectroscopy (Siebenmorgen et al. 2005; Hao et al. 2005; Spoon et al. 2007; Schweitzer et al. 2008; Mendoza-Castrejón et al. 2015; Hatziminaoglou et al. 2015) as well as high spatial resolution ground-based data (Hönig et al. 2010; Alonso-Herrero et al. 2011; González-Martín et al. 2013; Esquej et al. 2014; Ramos Almeida et al. 2014a). Another challenge for these models is trying to explain the spatial extent of the mid-IR emission which also looked puzzling initially. A significant part of the mid-IR emission appears to come from the ionization cones (Braatz et al. 1993; Cameron et al. 1993; Hönig et al. 2012, 2013), which suggests the presence of dust in the ionization cones either in the form of discrete clouds or as outflows (Efstathiou et al. 1995; Elitzur \& Shlosman 2006). The data suggest that what is needed might be a combination of smooth and clumpy structures in AGNs which may extend into the narrow line region, see Netzer (2015) for a recent review. The present picture is also supported by recent hydrodynamical simulations which predict a filamentary torus and a turbulent disk component (Schartmann et al. 2014).

Large ground-based telescopes offer an opportunity to better isolate the contribution of the host galaxy and circumnuclear star formation from the AGNs when compared to observations 
from space (Alonso-Herrero et al. 2011; Ramos Almeida et al. 2011; Asmus et al. 2014). Spitzer spectra provide a resolution of $\sim 3^{\prime \prime}$, whereas high resolution mid-IR spectra from the ground are an order of magnitude sharper, and sensitive enough to observe local Seyferts at luminosities $<10^{46} \mathrm{erg} / \mathrm{s}$. Such high resolution studies have been undertaken for example in NGC 1068, NGC 1365, NGC 3281, and Circinus (Mason et al. 2012; Roche et al. 2006; Alonso-Herrero et al. 2012; Sales et al. 2011; Esquej et al. 2014). A sample of 19 Seyferts are presented by Hönig et al. (2010) and another 22 Seyferts are studied by González-Martín et al. (2013). At $\sim 20$ pc resolution Ruschel-Dutra et al. (2014) detected the silicate feature at $9.7 \mu \mathrm{m}$ in absorption for the Seyfert 2 NGC 1386, and in emission for the Seyfert 1 NGC 7213 with a peak wavelength beyond $10.5 \mu \mathrm{m}$. Ramos Almeida et al. (2014a) compared the nuclear mid-IR spectrum with the Spitzer spectrum and find that the AGN in Mrk1066 dominates the continuum emission at $\lambda<15 \mu \mathrm{m}$ on scales of $\sim 60 \mathrm{pc}(90-100 \%)$, whereas this contribution decreases to below $50 \%$ when the emission of the central $\sim 830 \mathrm{pc}$ is considered.

Infrared interferometry offers unprecedented angular resolution that allows resolving the inner regions of dusty AGNs (Jaffe et al. 2004; Tristram et al. 2007, 2009). It has been shown that the inner torus radii derived from visibility curves as expected scale approximately with the square root of the AGN luminosity (Suganuma et al. 2006; Kishimoto et al. 2011), except for some deviations that are observed for fainter sources (Burtscher et al. 2013). A sample of nearby Seyfert galaxies observed with IR interferometers (Hönig et al. 2013) reveal a diversity of obscuring structures. A significant part of the mid-IR emission of certain sources appears to come from the polar direction. These observations are difficult to reconcile with torus models alone and challenges the justification of using clumpy models to fit that emission (Hönig et al. 2013). Polar dust may be physically disconnected from the torus and may arise from a radiatively driven dusty wind blown off the inner region of the torus.

ALMA observations have recently been reported for the Seyferts NGC 1566 (Combes et al. 2014), NGC 1068 (García-Burillo et al. 2014) and NGC 34 (Xu et al. 2014). Typically at $25-50 \mathrm{pc}$ resolution an unresolved core and a circumnuclear disk extending about $200 \mathrm{pc}$ in radius is detected.

Solutions of the radiative transfer in clumpy AGN tori employ methods that have different levels of sophistication. A first formalism was presented by Nenkova et al. (2002) utilizing a $1 \mathrm{~d}$ radiative transfer code and computed SED of clouds by assuming a slab geometry. The slabs are heated from different angles and then averages are taken so that they mimic the emission of spheres. These spheres are arranged so that a torus-like geometry is simulated. Hönig et al. (2006) pre-computed these clouds in two-dimensions and Hönig \& Kishimoto (2010) with an upgrade of that model in three-dimensions. In the AGN torus there are clouds that are not illuminated directly by the central source because other optically thick clouds lie along the line of sight towards the primary heating source. Such clouds are shadowed and indirectly heated. In the above mentioned models it is assumed that this indirect heating is coming from clouds in the immediate surrounding which are directly heated so that averages can be build. Clumps, which are directly heated by photons reflected on a surface of an optically thick disk were not treated. Clumpy torus models are successful in explaining the strengths of the silicate emission and absorption band. However, they do not account for very deep silicate absorptions and under-predict the near-IR emission (Nenkova et al. 2010; Spoon et al. 2007). Self-consistent three-dimensional (3D)

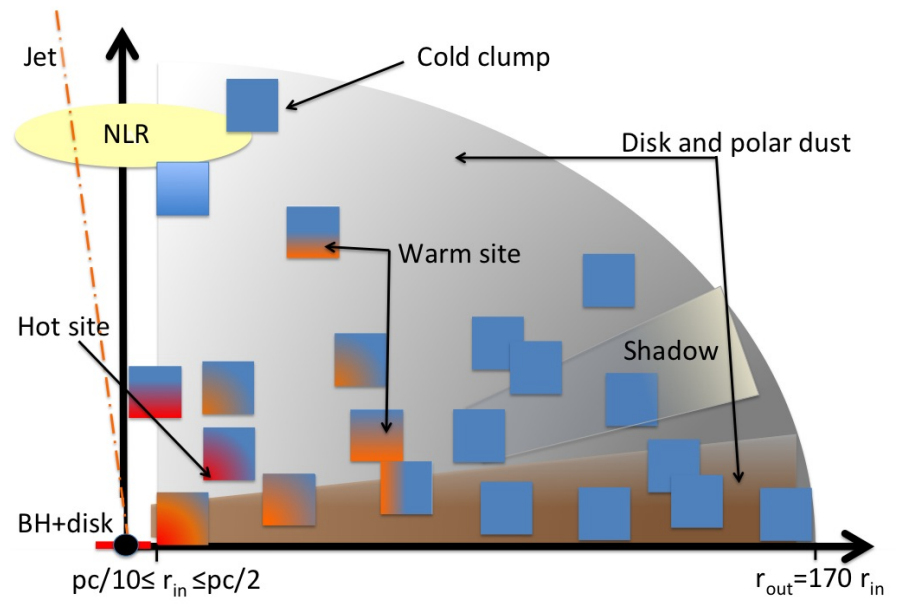

Fig. 1. Schematic view of the AGN structure assumed in this work. Dust extends from the dust evaporation radius at $\mathrm{pc} / 10 \leq r_{\text {in }} \leq \mathrm{pc} / 2$ up to distance of $r_{\text {out }}=170 \times r_{\text {in }}$ from the black-hole $(\mathrm{BH})$. We note that dust from the smooth disk emerges also as a diffuse component into the polar region. Dust clouds are pc sized with cube length of $d=3 \times r_{\text {in }}$. The hot, warm and cold side of the clouds is indicated and for one clump their shadows. The accretion disk in the immediate black-hole environment and the jet is not treated.

clumpy torus models are presented by Schartmann et al. (2008) and Stalevski et al. (2012a). Mendoza-Castrejón et al. (2015) find that Seyfert galaxies have clumpy dust distributions and/or a disk component. The formation of a clumpy and a continuous dust component is also predicted by hydrodynamical simulations (Schartmann et al. 2014).

In this paper we describe our SED library of two-phase AGN torus models that has been computed with the fully selfconsistent 3D Monte Carlo radiative transfer code of Heymann \& Siebenmorgen (2012). In addition to the clumps, we also apply a density distribution of a disk component that extends into the ionization cones. We will show below that the emission from such diffuse dust in the ionization cones can dominate the total emission from an edge-on view. This can explain why some Seyferts show extended emission coincident with the ionization cones at $10 \mu \mathrm{m}$ although there is an optically thick torus which lies in a plane perpendicular to the ionization cones. The emission coming from the ionization ones is also much more compact than the torus in agreement with interferometric observations (Burtscher et al. 2013; Hönig et al. 2013). We discuss the general properties of the models (Sect. 3) and apply the SED library to fit a number of representative objects (Sect. 4). We summarize our findings in the conclusions (Sect.5).

\section{AGN Model}

In this section we describe the AGN model library of SEDs. We outline the radiation transfer code, the assumed dust structure, and the library set-up. We choose a condensed description of the AGN model with all parameters kept constant except a minimum of five key parameters that are varied to compute the library. We visualize the dust density distribution and AGN images at different wavelengths, and show the impact of our free parameters on the SED.

Most models for the torus in AGNs consider dust that is representative for the diffuse interstellar medium and the fact that there are huge differences in the density and the radiation environment of both media is ignored. Our library is computed 
considering fluffy grains. The rational for this and the dust properties assumed are discussed in Sect. 2.6. Finally, we discuss the impact of scattering in spatially unresolved observations of the extinction curve in AGNs.

\subsection{Radiative transfer}

For our calculations we apply the Monte Carlo (MC) continuum radiative transfer code of Heymann \& Siebenmorgen (2012). The code allows us to follow the light path of photons that are emitted from a heating source through an arbitrary 3D dust configuration. Photons are binned into packets of equal energy, those photons of one packet have the same frequency. Dust absorption, scattering and emission events are computed by chance. The process depends on the optical depth and the absorption and scattering cross section of the dust. We utilize the MC algorithm developed by Bjorkman \& Wood (2001), which is iterative-free as it employs an immediate update of the dust temperatures. For very optically thin regions we use the method by Lucy (1999) which reduces uncertainties in the dust temperatures. Anisotropic scattering is treated assuming the phase function $p(\cos (\theta))$ as given by (Henyey \& Greenstein 1941)

$$
p(\cos (\theta))=\frac{1}{4 \pi} \frac{1-g^{2}}{\left(1+g^{2}-2 g \cos (\theta)\right)^{3 / 2}},
$$

where $\theta$ is the scattering angle. The anisotropy factor $g$ of the grains is provided by the dust model and whenever $g=0$ we consider isotropic scattering.

The model space is set up as cubes in an orthogonal Cartesian grid. Each cube can be divided into an arbitrary number of sub-cubes with constant density. An illustration of the grid is given in Fig. 1 by Heymann \& Siebenmorgen (2012). The subdivision of cubes allows a finer sampling whenever required. The advantage of such a grid is that it leads to a huge reduction of the computer memory for some applications. Examples of this are the interface of optically thick clumps that are embedded in an otherwise homogeneous medium, regions close to the dust evaporation zone or configurations where the ratio of the outer and inner radius from the central source where dust exists is large $r_{\text {out }} / r_{\text {in }}>100$. The number of cells can be reduced further by a factor of eight by locating the source at origin and assuming mirror-symmetry with respect to the planes at $x=0$, $y=0$ and $z=0$. Vectoring the code so that it can run on shared memory devices reduces the huge computing time of the MC procedure. The number of photon packets that can be followed in parallel on such machines scales roughly with the number of processing units available and reduces the required computing time accordingly.

Ray-tracing in parallel projection from the detector of the observer through the model space, using temperatures and scattering events for each cell of the MC run, allows the generation of high spatial resolution and high signal-to-noise images. In this work we derive the SED of the object by counting the photon packets that eventually escape the model space into a particular solid angle. This method gives within the photon noise the same results when compared to SEDs computed by ray tracing. The later method has the advantage that it provides images for each frequency. However, it is expensive in computing time. For one AGN model and SEDs computed in 9 viewing direction the ray tracer takes about half a day of computing time while the photon counting is completed in a few seconds.

\subsection{AGN Structure}

The super-massive central black hole with its accretion disk (Shakura \& Sunyaev 1976) is considered to be the primary heating source of the circumnuclear environment. It emits anisotropic hard UV and optical photons (Netzer 1987; Kawaguchi \& Mori 2011). The anisotropy of the primary source does not have a strong impact on the torus spectrum when compared to models with isotropic heating. The overall SED shape and the $10 \mu \mathrm{m} \mathrm{Si-O} \mathrm{dust} \mathrm{feature} \mathrm{are} \mathrm{not} \mathrm{seriously} \mathrm{affected} \mathrm{by} \mathrm{the}$ anisotropy (Stalevski et al. 2012a). Therefore the emission of the primary heating source is taken to be isotropic. In optically thick applications the spectral shape of the heating source has virtually no impact on the dust emission spectrum. We assume that the primary energy source takes a spectral shape approximated by Rowan-Robinson (1995):

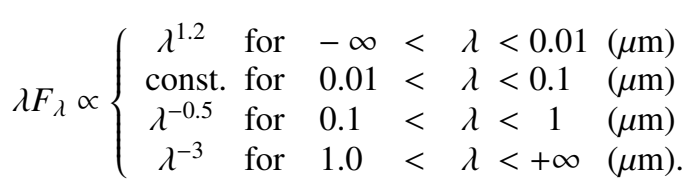

The inner radius $r_{\text {in }}$ of the dust torus is set close to the dust evaporation region, which scales with the total AGN luminosity as $L_{\mathrm{AGN}} \propto r_{\text {in }}^{2}$. For type $1 \mathrm{AGNs}$ this relation is confirmed by reverberation measurements and interferometry (Kishimoto et al. 2011). As long as this relation is respected, the SED becomes scale invariant, so that the SED shape does not vary for different AGN luminosities. We therefore keep the luminosity of the primary source constant at $L_{11}=10^{11} L_{\odot}$. The sublimation temperature of the dust depends on details such as grain mineralogy, porosity and size. We assume that it is somewhere between $800-1800 \mathrm{~K}$ and use $r_{\text {in }}$ as a free-parameter.

The required maximum size of the torus depends on the observed wavelength. However, choosing $r_{\text {out }}$ smaller than the size corresponding to the temperature, respectively wavelength, of interest results in an artificial cut-off in the brightness distribution of the models. For example, when selecting $r_{\text {out }} / r_{\text {in }} \sim 25$ the cut-off occurs at temperatures of $\sim 400 \mathrm{~K}$, which corresponds to wavelengths around $8 \mu \mathrm{m}$. It is therefore important to select $r_{\text {out }}$ large enough for all cases to avoid artificial cut-off in the brightness distribution (Hönig \& Kishimoto 2010). We assume that the dust density in the torus declines with distance from the AGN, so that at some point a further increase in $r_{\text {out }}$ has no impact on the SED. We find $r_{\text {out }} / r_{\text {in }} \sim 170$ is a reasonable choice. This is larger than that assumed in most of the previous AGN models.

The distribution of the dust in AGNs is still a matter of debate. It was realized early on that the distribution of gas and dust is most likely clumpy and in a torus-like structure (Krolik \& Begelman 1988). To account for IR observations and the $10 \mu \mathrm{m}$ silicate feature radiative transfer calculations of clumpy dust distributions have been carried out, e.g. (Nenkova et al. 2002; Schartmann et al. 2008; Heymann \& Siebenmorgen 2012; Stalevski et al. 2012a). Dust models that assume homogeneous or smooth distributions (Pier \& Krolik 1992; Efstathiou \& Rowan-Robinson 1995; Efstathiou et al. 2014) have also been considered. AGN models assuming clumpy or smooth dust distributions can produce similar SEDs (Feltre et al. 2012).

Interferometric observations of nearby AGNs have so far resolved disk-like or extended dust emission components (usually coincident with the ionization cones) down to pc scale. Unresolved structures such as clumps are below the accessible resolution limits of $\sim 15$ mas (Tristram et al. 2014). X-ray monitoring of discrete absorption eclipse events of Seyferts is presented by Markowitz et al. (2014). They find a higher detection 
probability of clumps for type 2 than for type 1 , and from the observed column density an optical depth of the clumps between $\tau_{\mathrm{V}}=20-160^{1}$ is estimated. The current data of the X-ray study provides insight into the cloud distribution only close to the dust evaporation zone. For type 2 objects the X-ray absorption can also be explained by a homogeneous medium.

We consider a dust structure in the AGN which is approximated by an isothermal disk that is embedded in a clumpy medium. The midplane of the disk is at $z=0$, so in the $x y$-plane of the coordinate system the dust density distribution of the disk is given by

$\rho(x, y, z)=\rho_{o} \frac{r_{\text {out }}}{r} \mathrm{e}^{-\pi\left(\frac{z}{2 \mathrm{~h}}\right)^{2}}$.

We choose $\rho_{o}$ so that the optical depth of the disk midplane in the $V$ band, which is measured between $r_{\text {in }}$ and $r_{\text {out }}$, is $\tau_{V \text {,mid }}=$ $0,30,100,300$ or 1000 . The scale height is approximated by (Pascucci et al. 2004)

$h=\frac{r_{\text {out }}}{8}\left(\frac{2 r}{r_{\text {out }}}\right)^{1.125}$.

In addition there are a number $N_{\mathrm{cl}}$ of clouds that are passively heated from outside. The possibility that massive stars heat clouds from inside is not treated. The density of the clouds is assumed to be constant. This is consistent with the small scatter in the column density of the clouds as derived from X-ray observations (Markowitz et al. 2014). The clouds are arbitrarily distributed in the model space. We assume, for random numbers denoted by $z$, that the clouds have an isotropic distribution along the azimuth angle $\phi=\pi / 2 \xi$, but they are weighted along the polar angle $\theta$ by $\cos (\theta)=3^{2}$, so that there are more clumps at low than at high latitudes, and along the radial direction $r$ an equidistant separation with the disk midplane given by

$r=r_{\text {in }}+\sqrt{3\left(r_{\text {out }}^{2}-r_{\text {in }}^{2}\right)}$.

A schematic of the AGN model is shown in Fig. 1. The potential accretion disk in the immediate black-hole environment, where dust cannot survive, and the jet emission is not treated in our model. The dust disk extends from a radius of less than 1 up to a few hundred pc distance from the primary heating source. We assume dust clouds have a cube size of $0.3-3 \mathrm{pc}$. The hot, warm and cold side of these clumps and the shadowing caused by them is computed self-consistently in the 3D radiative transfer model. We note that all scales correspond to an AGN luminosity of $10^{11} L_{\odot}$. As discussed above all radii scale as $L^{1 / 2}$.

\subsection{Technical set-up}

The model grid is set up into $(51,51,51)$ basic cubes with a side length of a cube $d$ depending on the choice of the inner dust radius. It is given by $d=3 . \overline{3} r_{\text {in }}$. The central cube has $(900,900,900)$ subcubes, and along each direction the following $(10,10,10)$ basic cubes are each subdivided into $(15,15,15)$ subcubes. We can achieve a resolution of $4 \times 10^{14} \mathrm{~cm}$ in the inner part of the torus, whereas by comparison the resolution in the hydrodynamical simulations of Schartmann et al. (2014) was $4 \times 10^{17} \mathrm{~cm}$. Each cloud is divided into $(3,3,3)$ basic cubes and each of these is divided into $(15,15,15)$ sub-cubes. This gives a total of more than 90000 cells for each clump, which ensures that even for the highest optical depth cases each sub-cube of the

\footnotetext{
1 Unless otherwise stated the optical depth is specified for the $V$ band.
}
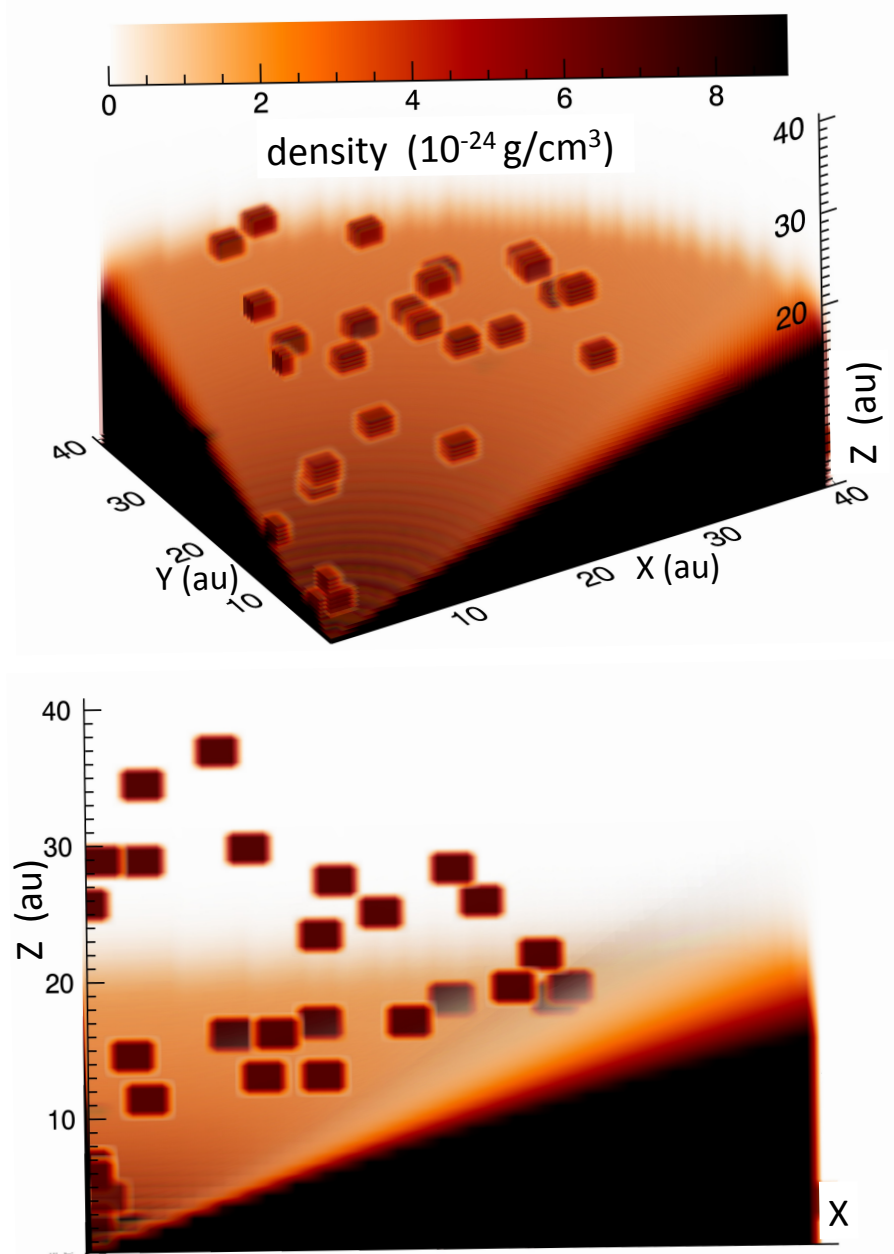

Fig. 2. Visualization of the dust density distribution of an octant of the AGN model space. A view from high latitudes (top) and edge-on along the $x, z$ axis (bottom) is shown. Clumps deep inside the disk are not visible. We note the presence of clumps in the polar region. The axes are in arbitrary units.

clump has an optical depth along its axis of $\tau_{V} \lesssim 1$. This criterion is similar to the one used by Hönig et al. (2006) who demonstrated that the cell temperatures converge in that case (see their Table 1). Stalevski et al. (2012a) used clouds with an optical depth $\tau_{V} \gtrsim 100$. These clumps are divided into $(8,8,8)$ cells when the cloud number is low, whereas only a single cell is used when the number of clouds is high. The latter simplification is not justified whenever the optical depth of a sub-cube of a cloud becomes larger than 1 . SEDs computed for optically thick clouds and ignoring sufficient sub-sampling drastically underestimate the IR emission.

The AGN spectrum (Eq. (2)) is divided into 256 frequency bins and for each bin 200000 photon packets are emitted. In our treatment this is sufficient to achieve a reasonable signal-to-noise of fluxes in the submillimeter. SEDs are computed in different viewing angles that are equally spaced in $\cos \theta$ between $0^{\circ}$ and $90^{\circ}$. The SEDs are reasonably well sampled by a division into nine different viewing angles.

\subsection{Library setup}

When setting up the SED library of AGNs we need in addition to the viewing angle four key parameters: (1) the inner radius $r_{\text {in }}$, 
Table 1. Parameters of the AGN library.

\begin{tabular}{lll}
\hline \hline Parameter & Symbol & Values \\
\hline Viewing angle $^{a}$ & $\theta\left(^{\circ}\right)$ & $\begin{array}{l}86,80,73,67,60, \\
52,43,33,19\end{array}$ \\
\hline Inner radius $^{b}$ & $r_{\text {in }}\left(10^{17} \mathrm{~cm}\right)$ & $3,5.1,7.7,10,15.5$ \\
\hline $\begin{array}{l}\text { Cloud volume } \\
\text { filling factor }\end{array}$ & $\eta(\%)$ & $1.5,7.7,38.5,77.7$ \\
\hline Cloud optical depth $^{d}$ & $\tau_{V, \text { cl }}$ & $0,4.5,13.5,45$ \\
\hline $\begin{array}{l}\text { Optical depth of } \\
\text { disk midplane }\end{array}$ & $\tau_{V, \text { mid }}$ & $0,30,100,300,1000$ \\
\hline
\end{tabular}

Notes. ${ }^{(a)}$ The viewing angle is equally binned in $\cos (\theta)$ and measured from the $z$-axis to the $x, y$ plane. ${ }^{(b)}$ The luminosity of the primary source is kept constant at $L_{11}=10^{11} L_{\odot}$. ${ }^{(c)}$ The cloud volume fraction corresponds to the number of clouds of $N_{\mathrm{cl}}=160,800,4000,8000$ within the 3D model space. ${ }^{(d)}$ The cloud optical depth is measured along the edge of the cloud that has a structure of a cube. ${ }^{(e)}$ The disk midplane is located in the $x, y$-plane at $z=0$. We measure $\tau_{V \text {,mid }}$ along the midplane from the source to the outer edge of the disk.

(2) the volume filling factor of the clouds $\eta$, (3) the optical depth of the clouds $\tau_{V, \mathrm{cl}}$, and (4) the optical depth of the disk midplane $\tau_{V \text {,mid }}$. There are other parameters, such as the outer radius, $r_{\text {out }}=$ $170 \times r_{\text {in }}$, up to where dust exists, details of the clump structure (Sect. 2.2) or their distribution function (Eq. (5)), which are all kept constant. The variations of the free parameters of the SED library are summarized in Table 1 . In the AGN library there are 400 models each providing SEDs for nine viewing directions.

We varied the density profile within a cloud, e.g. following a $1 / r$ profile, and experimented with the cloud structure, for example using spherical clumps instead of cubes. We confirm the results of Hönig \& Kishimoto (2010), who concluded that unless very peculiar clump surfaces are used, the exact shape of the clumps does not matter too much and a spherical or cubic representation probably catches the essence of the dust clouds in AGN tori. These authors find somewhat redder mid-IR colors when distributing more dust clouds at larger distances. We also varied the cloud distribution along the radial direction and the sizes of the clouds. We also experimented with using a large number of small clouds in the inner region and a small number of large clouds in the outer region. We compared models of the same total cloud mass and found that all of these changes of the cloud properties and distribution functions has some but no significant impact on the SED. We therefore do not consider variation of these properties in the library.

Finally, we implemented also clumps with parameters as derived from X-ray eclipse observations (Markowitz et al. 2014). Such clumps are detected in the central region of the AGN, so in our model in the innermost cube. They are assumed to be as small as $3 . \overline{3} \times 10^{14} \mathrm{~cm}$ and have $\tau_{\mathrm{V}}=20-160$. For these $\mathrm{X}$-ray clumps we computed a single clump temperature. We find that clumps detected by X-ray eclipse events have marginal impact on the SED as long as their number stays below several million. Therefore we neglect such X-ray clumps in the rest of the paper.

\subsection{Visualization}

As an aid in visualizing the AGN structure we show the dust density distribution and images at different wavelengths. The particular parameters of the AGN model we visualize are $r_{\text {in }}=0.25 \mathrm{pc}$, $\tau_{V, \mathrm{cl}}=4.5, \tau_{V \text {, mid }}=1000, \eta=1.5 \%$. A $3 \mathrm{D}$ visualization of the dust density distribution for an octant of the model space as seen from high latitudes and in edge-on view is shown in Fig. 2. The density inside the disk is so high that clumps are only visible above or within the upper surface layer of the disk.

Images of the same AGN model are shown in Fig. 3 for different viewing directions and wavelengths. The image at $1 \mu \mathrm{m}$ is dominated by scattered light with an exception in the face-on view where a small contribution by dust emission is visible in the innermost region. In scattered light only the upper and lower surfaces of the disk are visible. The optical depth is too high for the photons to penetrate deeper into the disk. This explains why there are two distinct gray areas noticeable. Clumps are visible as the few bright dots in the middle and bottom panel (left). The image at $10 \mu \mathrm{m}$ displays emission by warm dust and scattering is no longer important. The optical depth becomes smaller and one probes deeper inside the disk (compare top-left and topmiddle panel of Fig. 3). The disk itself remains optically thick at $10 \mu \mathrm{m}$ so that two distinct surfaces are visible as in the scattering light images. As can be seen in the top-middle panel, the emission from the diffuse dust in the ionization cones can dominate the total emission from an edge-on view. This can explain why some Seyferts show extended emission coincident with the ionization cones at $10 \mu \mathrm{m}$ although there is an optically thick torus which lies in a plane perpendicular to the ionization cones. The image at $10 \mu \mathrm{m}$ shows also the shadowing of the clumps. They can be noticed as notched contours or jet-like structures (top-middle). Behind a clump there is less direct heating from the central source, the dust becomes colder and the $10 \mu \mathrm{m}$ flux is reduced. The image at $100 \mu \mathrm{m}$ reveals the cold dust emission and at this wavelength the disk is optically thin and the radiation appears isotropic.

The size of the torus becomes wavelength dependent if one cuts the image contrast at higher flux levels, e.g. take $\log \left(F / F_{\max }\right)>-4$ in Fig. 3 (Stalevski et al. 2012b). At shorter wavelengths radiation from the inner region dominates and at longer wavelengths, the emission arises from cold dust deeper inside the disk and from farther out.

\subsection{AGN dust properties}

Dust in dense environments such as in the circumnuclear regions of AGNs is certainly different from the dust in the diffuse interstellar medium (ISM). The variation is caused by the huge difference in the density in both media and for AGNs some observational evidence of a change in the dust properties is presented by Maiolino et al. (2001). The basic process for this modification of the dust is probably grain coagulation which leads to fluffy particles and grain growth (Mathis \& Whiffen 1989).

The $2175 \AA$ extinction bump, which is attributed to small graphites and PAHs, is not detected in AGNs (Maiolino et al. 2001). The lack of PAH emission in the proximity of the AGN was used as an indication that particles smaller than $10 \mathrm{~nm}$ are destroyed (Voit 1992; Mason et al. 2007). However, Alonso-Herrero et al. (2014) and Ramos Almeida et al. (2014b) detected in 6 Seyfert galaxies the $11.3 \mu \mathrm{m}$ PAH feature in the nuclear region. They show that PAH molecules can survive in the nuclear environments of low luminosity AGNs as close as $10 \mathrm{pc}$ from the central source. They interpret this as evidence that the PAHs are not destroyed in the torus by the hard radiation of the AGN.

Inner radii derived from $K$-band reverberation mapping of type 1 AGNs are a factor of 3 smaller than what is expected from standard ISM dust. Kishimoto et al. (2007) suggest that 

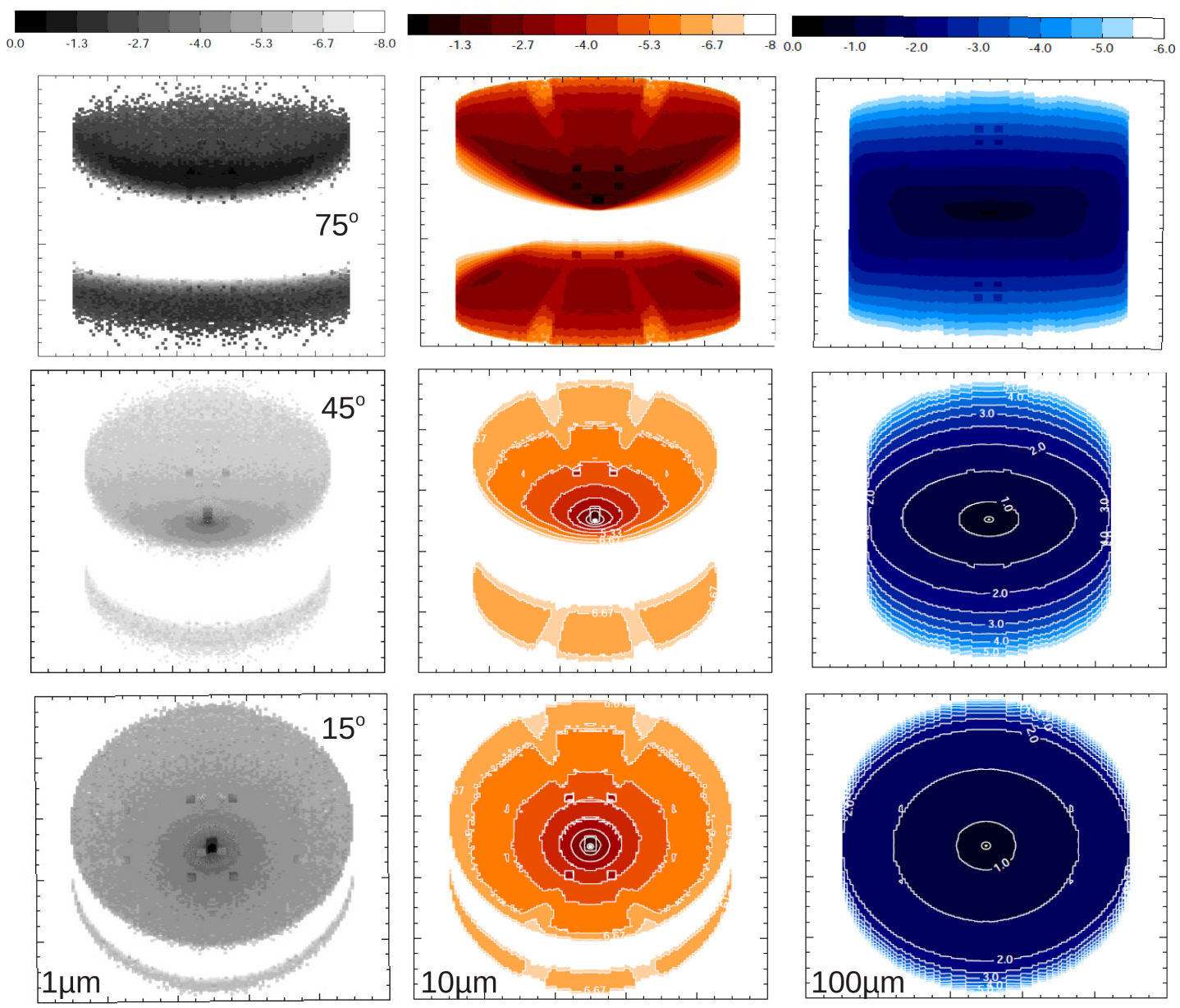

Fig. 3. Images of the AGN torus computed by ray-tracing at viewing directions corresponding to $15^{\circ}$ (face-on, bottom), $45^{\circ}$ (middle) and $75^{\circ}$ (edge-on, top) and from left to right at wavelengths of 1 (gray), 10 (orange), and $100 \mu \mathrm{m}$ (blue). The images are logarithmically scaled and at each wavelength the flux is normalized to its peak value.

this is due to the fact that the grains are larger than standard ISM dust. However, Hönig \& Kishimoto (2010) conclude that the grain size distribution in AGNs is the same as that usually adopted for the ISM. Previous models of AGN tori assumed diffuse ISM dust, graphite dominated dust, and dust with different optical constants than usually assumed for ISM dust. Fritz et al. (2006) shows that variations of the silicate feature strength may be explained by changing the optical constants of the dust or its distribution in the torus.

In this study we consider fluffy mixtures of silicate and amorphous carbon grains (Krügel \& Siebenmorgen 1994). Optical constants of the materials are from Zubko et al. (1996) and Draine (2003), respectively. The bulk density of the dust materials is $2.5 \mathrm{~g} / \mathrm{cm}^{3}$, the carbon-to-silicate abundance ratio is 6.5 , and $50 \%$ of the volume fraction of fluffy particles is vacuum. We consider large particles with radii $a$ between 16 and $260 \mathrm{~nm}$. They follow a grain size distribution with number density $\propto a^{-3.5}$ (Mathis et al. 1977). The influence of the dust cross section on the AGN emission is exemplified in Fig. 4. For comparison we apply dust cross sections of pure (unmixed) particles that we call ISM dust (Siebenmorgen et al. 2014). In addition to the grain structure, ISM grains are otherwise identical to fluffy grains. They have the same optical constants, abundances, and sizes. Fluffy grains are more efficient absorbers and have lower scattering cross sections than ISM dust for the whole spectrum. The extinction cross section of fluffy grains in the $V$ band is $K_{V}=33.3\left(\mathrm{~g}\right.$-dust $\left./ \mathrm{cm}^{3}\right)$. This is a factor of 2 larger than for
ISM dust. At $1 \mathrm{~mm}$ the cross section of fluffy grains is 12 times that of ISM dust. For the purpose of a comparison we take an AGN library model, computed using fluffy grains, inner radius $r_{\text {in }}=0.25 \mathrm{pc}$, cloud filling factor $\eta=1.5 \%$, cloud optical depth $\tau_{V, \mathrm{cl}}=4.5$, and optical depth of the disk midplane of $\tau_{V \text {,mid }}=1000$. A model with the same dust density distribution is run assuming ISM dust. For the latter model the disk midplane optical depth reduces to $\tau_{V \text {,mid }}=750$ and the optical depth of the clouds to $\tau_{V, \mathrm{cl}}=3.375$. Fluffy grains emit more strongly in the far-infrared (far-IR) and submillimeter than ISM dust and the SED peak wavelength shifts towards longer wavelengths. The cross-over wavelength where the AGN emission in face-on and edge-on views is identical, in other words the wavelength where the torus emission becomes isotropic, occurs for fluffy grains in the submillimeter $(\sim 200 \mu \mathrm{m})$ and for ISM dust in the far-IR $(\sim 100 \mu \mathrm{m})$.

In face-on view the emission in the near- and mid-IR is similar, whereas in edge-on view the small difference in the extinction of both dust models leads to a significant change in the continuum flux. In the silicate band at $10 \mu \mathrm{m}$ the fluffy grains have a shallower and broader absorption cross section when compared to ISM dust. This is also reflected in the SED that is shown in Fig. 4. In edge-on view the silicate absorption is noticed using ISM dust and not when fluffy grains are considered. In face-on view the silicate feature has a peak emission at $10.4 \mu \mathrm{m}$ for ISM dust and at $11.5 \mu \mathrm{m}$ for fluffy grains. In the dense dust environment of the AGN the particles have a higher sticking probability 


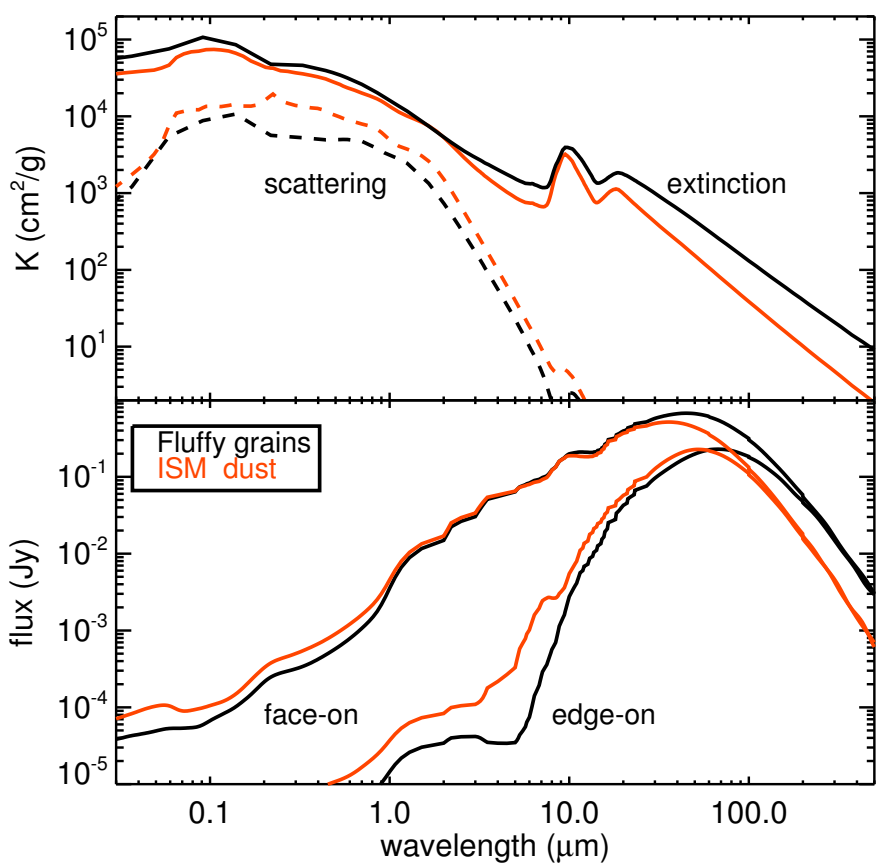

Fig. 4. Extinction (full line) and scattering (dashed) cross section $K$ as a function of wavelength (top). We show fluffy aggregates (black) of silicates and amorphous carbon (Krügel \& Siebenmorgen 1994) and identical materials as unmixed bulk particles (red) of the diffuse ISM (Siebenmorgen et al. 2014). For both dust models and with otherwise identical parameters the emission spectrum of a clumpy AGN torus is given for face-on and edge-on view (bottom).

than in the diffuse ISM and form rather fluffy dust agglomerates than dust in the diffuse ISM. The $2175 \AA$ extinction bump is not observed in AGN spectra and therefore we use in both dust models amorphous carbon that does not produce such a feature. In the scattering cross section ISM dust shows a feature at $0.2 \mu \mathrm{m}$ (Hofmeister et al. 2009) that is not present for fluffy grains. Such a scattering peak is not detected for AGNs.

\subsection{AGN extinction}

The fact that dust near AGN is different from the diffuse ISM may be best witnessed from changes in the extinction curve. However, we point out some observational difficulties. For unresolved observations, photon scattering within the beam may increase the detected flux altering the wavelength dependence of the extinction, and for clumpy media there are light paths towards the source at different optical depths. These effects are known to change the appearance of extinction curves and to account for this the effective optical depth is introduced (Natta \& Panagia 1984; Krügel 2009)

$\tau_{\text {eff }}=-\ln \frac{F_{\text {obs }}}{F_{\text {nd }}}$,

where $F_{\text {obs }}$ is the observed flux and $F_{\text {nd }}$ is the flux that would be observed in the absence of dust. Both quantities are easily derived by counting the number of photons within the aperture of an AGN model and comparing them to an identical MC run but without dust. We illustrate this for both dust models of Sect. 2.6 and take as the AGN model: $r_{\text {in }}=0.25 \mathrm{pc}, \tau_{V, \mathrm{cl}}=4.5$, $\tau_{V, \text { mid }}=1000, \eta=1.5 \%$, and in addition a disk-only model without clumps $(\eta=0)$. For these four models it was necessary to improve the photon statistics in the optical. We do this by launching 40 times more packets than for the SEDs computed for the library.

The effective extinction curves are displayed in Fig. 6 for three directions: face-on $\left(\theta \sim 33^{\circ}\right)$, an intermediate case with $\theta \sim 50^{\circ}$, and edge-on $\left(\theta \gtrsim 66^{\circ}\right)$. For reference we also show the extinction curve as derived from the dust cross section $K / K_{V}$ of the fluffy grains. In pencil-beam observations the effective extinction and the dust extinction are identical. It is striking that none of the effective extinction curves resembles the one of the dust model. There is no direct one-to-one link between the observed extinction curve and the wavelength dependence of the dust cross sections (Scicluna \& Siebenmorgen 2015).

In edge-on view $\tau_{V \text {,eff }}>0$ from the UV down to the near-IR. In the UV the extinction curve flattens, stays below the extinction of the dust model and becomes even gray in accordance with the findings by Natta \& Panagia (1984) and Wolf et al. (1998). From similar observational results it was claimed that the dust composition in the circumnuclear region of AGNs could be dominated by large grains (Maiolino et al. 2001). As demonstrated in Fig. 6 such a statement does not necessary hold. One cannot directly conclude that there is an increase in grain size when observing a flatter than usual extinction curve and this is especially the case when the object remains highly unresolved.

The effective extinction may also become negative. This occurs whenever there is more light detected with dust than without dust (Krügel 2009). Negative extinction can be measured when extra light is scattered into the beam and overshines the primary source. For intermediate or face-on views presented in Fig. 6 this occurs at wavelengths $\gtrsim 0.8 \mu \mathrm{m}$. In this case there is an optically thin view to the source and in addition light is scattered from the disk into the beam. In the face-on view of the disk-only model the enhanced scattering contribution is important already in the $V$ band so that $\tau_{V \text {,eff }}<0$. This explains the behavior of the dashed red line in Fig. 6 and remains stable as long as $\theta \lesssim 44^{\circ}$ (close to face-on). For edge-on views variations in $\theta$ are unimportant as long as the disk provides substantial extinction. For clumpy models and viewing angles that do not hit the disk $\left(\theta<60^{\circ}\right)$ small variations in $\theta$ have a large impact on $\tau_{V \text {,eff }}$ because by chance a clump may obscure the source or not.

The effects described above become more pronounced for ISM dust (dotted) than for the fluffy grains because the former have stronger scattering efficiencies (Fig. 4, top). They also produce significant steeper extinction curves than is expected from the dust model, in agreement with the qualitative estimates derived by Krügel (2009).

\section{Impact of parameter variation on the SED}

We illustrate in Fig. 5 changes in the SED when one parameter of the AGN library is varied while all the others stay fixed. For a particular model the SED in face-on view is shown in the top and in edge-on view in the bottom panel of Fig. 5. In both views, and respective panels, the SEDs are identical when the AGN emits isotropically. This happens at wavelengths long-ward of the far-IR peak flux. The AGN torus emits an-isotropically when the SED strongly depends on the viewing direction. This occurs at wavelengths short-ward of the far-IR peak. Panels a and b of Fig. 5 illustrate how an increase in the inner radius shifts the farIR peak to longer wavelengths. When the inner radius becomes larger the dust gets colder, hence shows a maximum emission at longer wavelengths so that in the far-IR and submillimeter the flux increases while in the near-IR the flux decreases.

Clumpy models without and with a homogeneous disk are shown in Figs. 5a, b, respectively. In face-on view models with a 


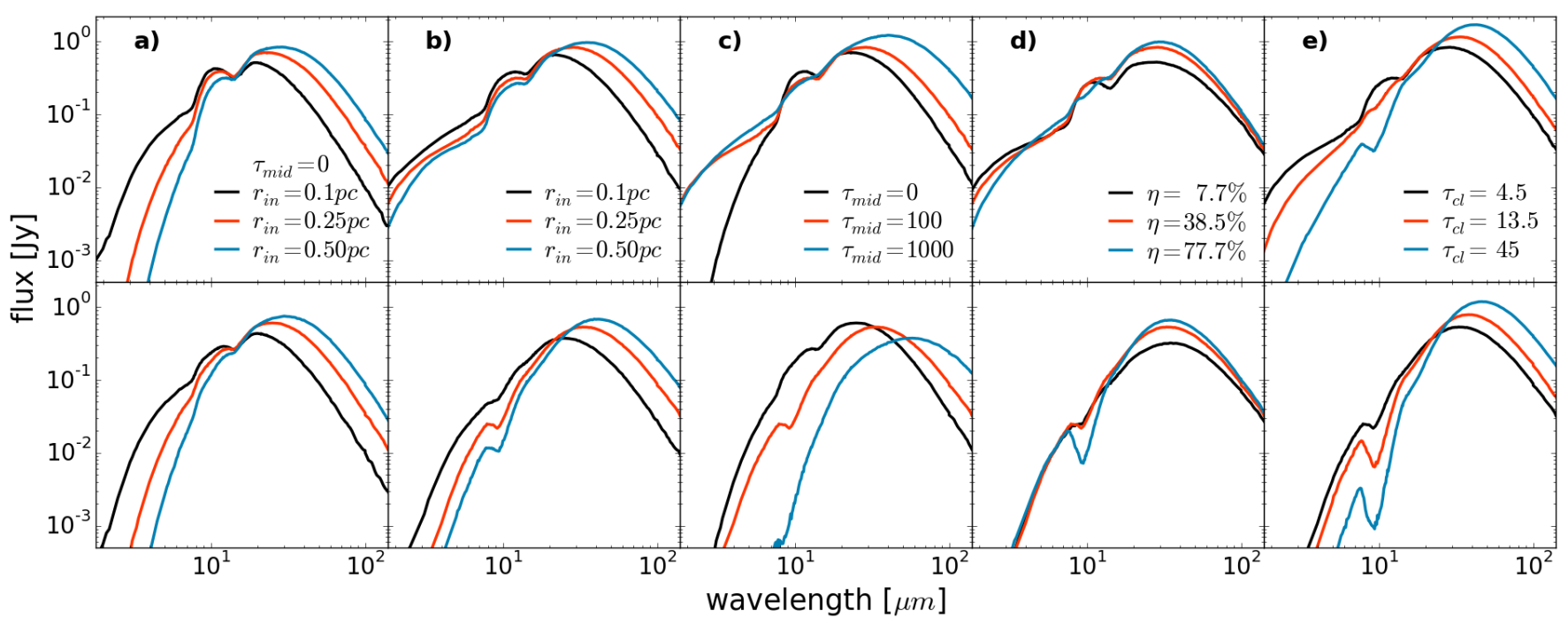

Fig. 5. Impact of AGN parameters on the SED in face-on (top) and edge-on (bottom) view. We vary the inner radius $r_{\text {in }}$ for models a) without and b) with a disk; c) the optical depth of the disk midplane $\left.\tau_{V, \text { mid }} ; \mathbf{d}\right)$ the volume fraction of the clouds $\eta$; and e) the optical depth of the clouds $\tau_{V, \text { cl }}$. Unless otherwise specified $r_{\text {in }}=0.25 \mathrm{pc}, \tau_{V, \text { mid }}=100, \eta=38.5 \%$, and $\tau_{V, \mathrm{cl}}=4.5$.

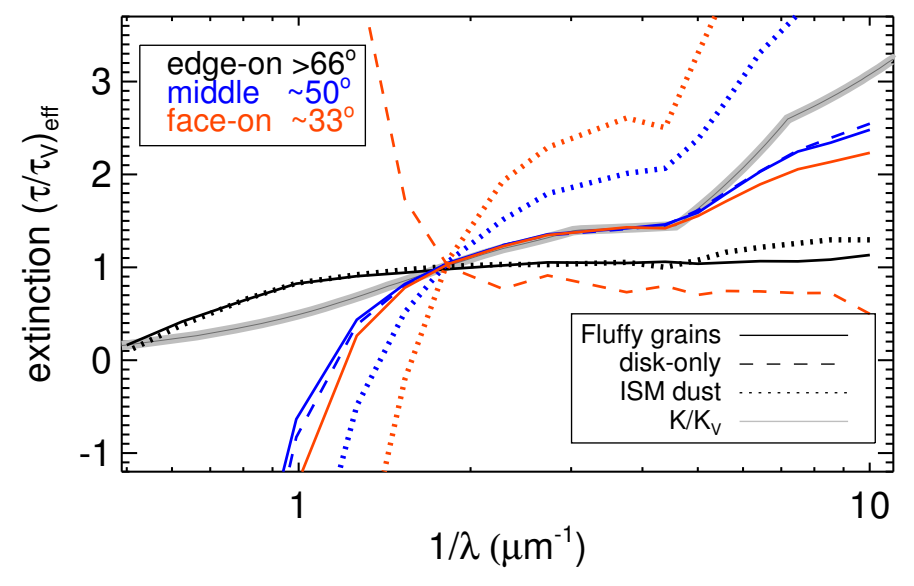

Fig. 6. Wavelength dependence of the effective extinction curves for three viewing directions: edge-on (black), intermediate (blue) and faceon (red) of AGN models as described in the text. The effective extinction curves of the AGN model computed with fluffy grains are shown as full line and for disk-only models as dashed lines, that of ISM dust as dotted lines. The extinction curve (thick gray) derived from the cross section of the fluffy grains is given for reference. All curves are normalized to the $V$ band.

disk show in the near-IR a huge flux increase when compared to models without a disk. The addition of disk material increases the total dust mass and this leads to an increase in the submillimeter flux at $200 \mu \mathrm{m}$ by a factor $2-3$. The impact of the midplane optical depth of the disk $\tau_{V \text {,mid }}$ is shown in Fig. $5 \mathrm{c}$. For edge-on views the disk provides additional dust extinction changing the silicate band from emission to absorption, while for face-on views the influence of the disk on the strength of that feature is small. When disk material is included there is additional hot dust in the system, and in face-on views the near-IR $(\sim 2 \mu \mathrm{m})$ flux increases by orders of magnitude (Fig. 5c, top). The near-IR flux may increase even further when a puffed-up inner rim, as known to exist in proto-planetary disks, is considered (Dullemond et al. 2002; Siebenmorgen \& Heymann 2012). The disk reduces the near-IR flux for edge-on views provided that the extinction is high enough $\left(\tau_{V \text {,mid }}>30\right.$, Fig. $5 \mathrm{c}$, bottom).
The optical depth of the clouds $\tau_{V \text {,cl }}$ also has a huge impact on the near-IR and submillimeter fluxes (Fig. 5e). In face-on view a change of the silicate band from emission to absorption is noticed when the optical depth of the clouds $\tau_{V \text {,cl }}$ is increased (Fig. 5e). For edge-on views an increase in $\eta$ will increase the dust extinction and hence produce a stronger $10 \mu \mathrm{m}$ absorption feature (Fig. 5d, bottom). The impact of parameter variation on the silicate band is further discussed in Sect. 3.1.

\subsection{Silicate feature}

Mid-IR spectra of AGNs show silicate features in absorption (Roche et al. 1991; Spoon et al. 2002; Siebenmorgen et al. 2004b; Levenson et al. 2007) or in emission (Siebenmorgen et al. 2005; Hao et al. 2005, 2007; Sturm et al. 2005; Sirocky et al. 2008). Hatziminaoglou et al. (2015) present a census of the silicate features in mid-IR spectra of 800 AGNs observed with Spitzer. Where the feature is in emission, in about $65 \%$ of the cases the peak wavelength is $>10.2 \mu \mathrm{m}$, whereas the shift of the $9.7 \mu \mathrm{m}$ absorption features is much smaller. The $10 \mu \mathrm{m}$ silicate emission and absorption features are also observed from the ground (Roche et al. 2006; Hönig et al. 2010; Alonso-Herrero et al. 2012; Sales et al. 2011; Esquej et al. 2014; González-Martín et al. 2013; Ramos Almeida et al. 2014b; Ruschel-Dutra et al. 2014), and it is noticed that the depth of the $10 \mu \mathrm{m}$ silicate absorption band is enhanced compared to that measured by Spitzer.

In the MC code we applied a fine wavelength sampling that allows us to discuss the behavior of the $10 \mu \mathrm{m}$ band. The dust cross section of the $10 \mu \mathrm{m}$ silicate band is larger than that of the $18 \mu \mathrm{m}$ feature (Fig. 4). The feature strength can be quantified similarly to the definition of Eq. (6) by its effective optical depth

$\tau_{\mathrm{Si}}=-\ln \left(\frac{F_{\text {peak }}}{F_{\text {cont }}}\right)$,

where $F_{\text {peak }}$ is the maximum or minimum of the observed inband flux at center wavelength $\lambda_{\mathrm{c}}$, and $F_{\text {cont }}$ is an estimate of the underlying continuum at that wavelength. We estimate the continuum for each model spectrum by a straight line with anchors 

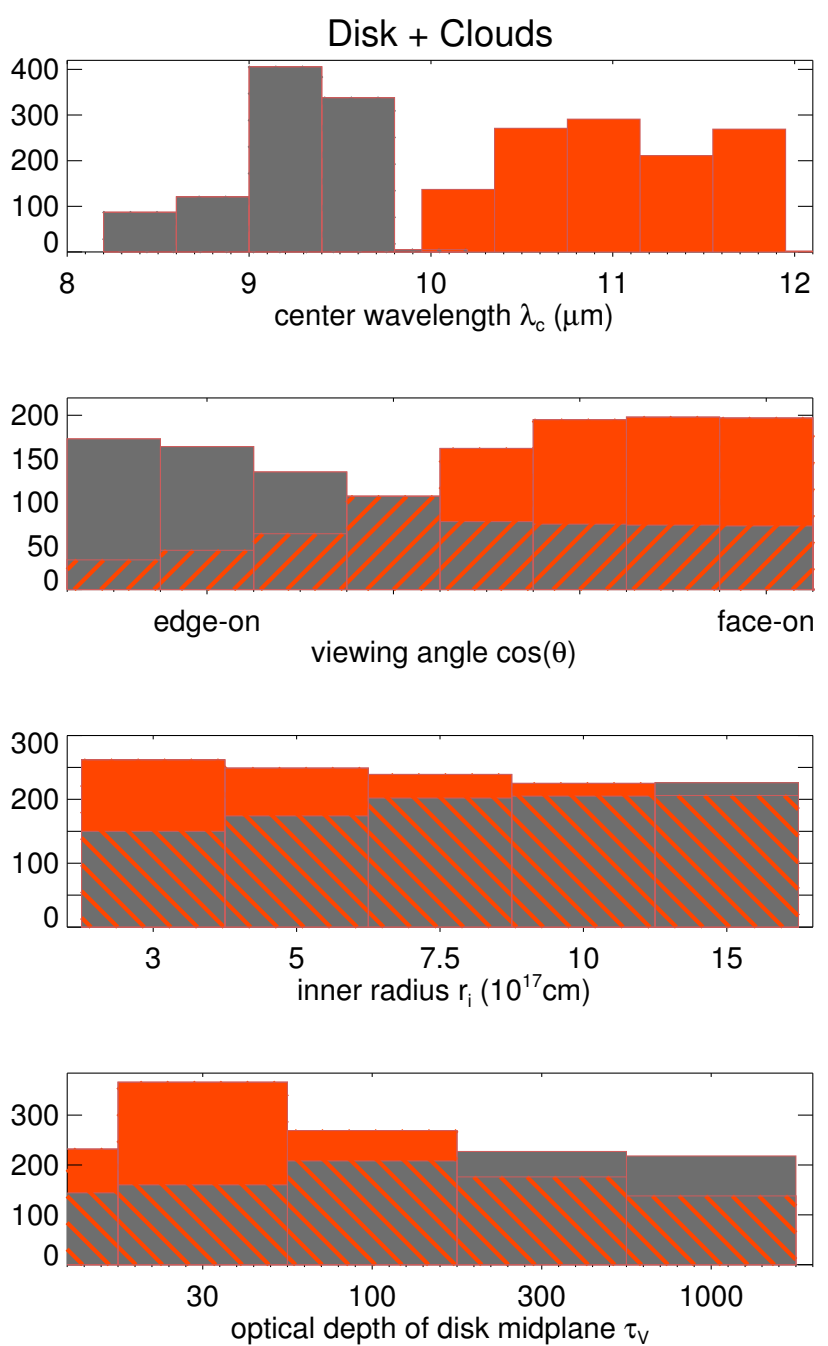
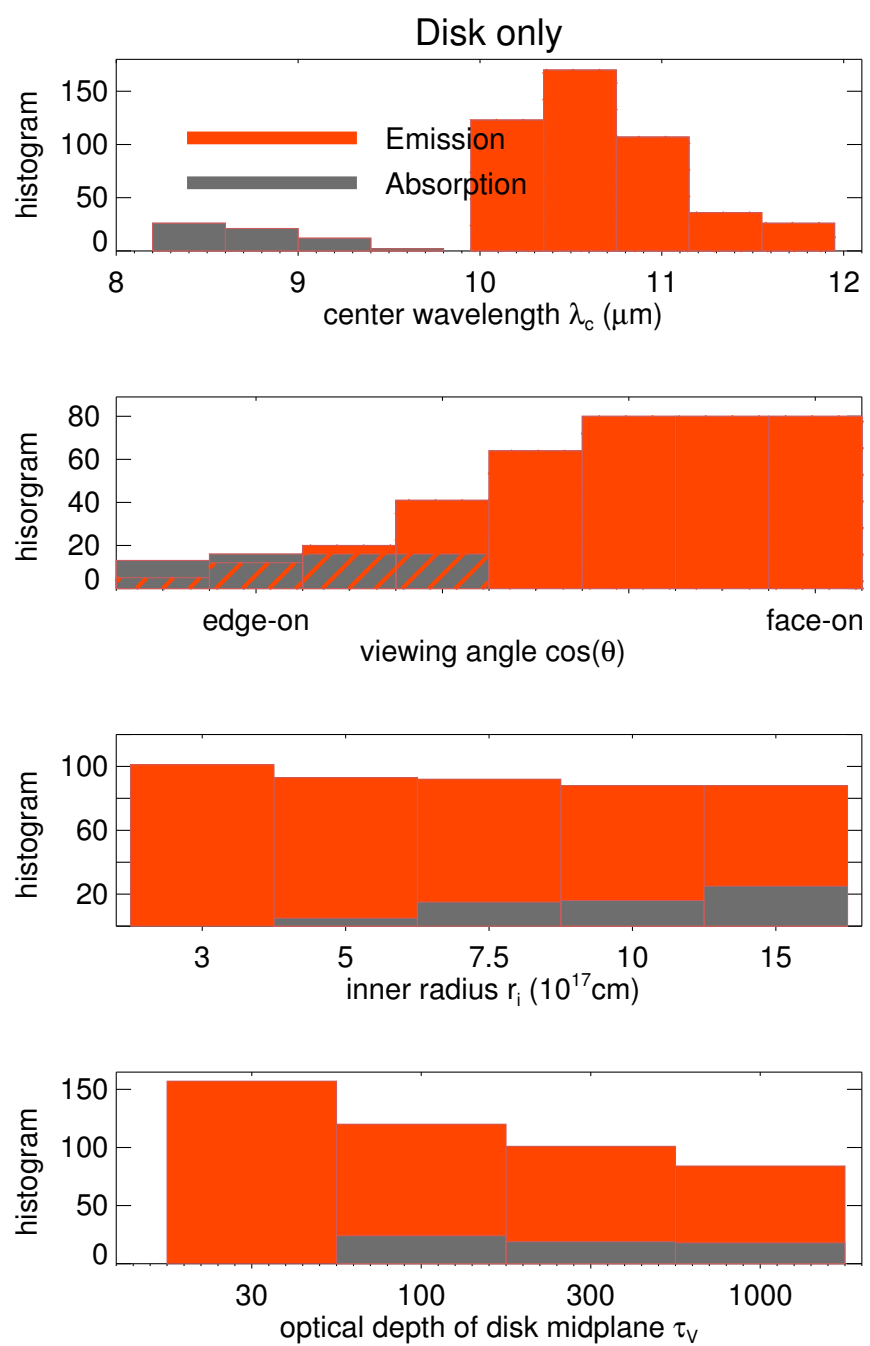

Fig. 7. Histograms of the silicate emission (red) and absorption (gray) band for the AGN library labeled "Disk+Clouds" (left) and disk-only models $($ right $)$. Distribution functions are shown from top to bottom for the central wavelength of the feature, the viewing angle, the inner radius of the AGN dust torus, and the optical depth along the midplane of the homogeneous disk.

set outside the red and blue wing of the band at wavelengths between $5-7 \mu \mathrm{m}$ and $12-14 \mu \mathrm{m}$. In this definition, emission features appear at $\tau_{\mathrm{Si}}<0$ and absorption features at $\tau_{\mathrm{Si}}>0$. Clumpy media offer some direct views to the inner hot regions that fill the absorption and reduce the depth of the band. The feature strength depends on the distribution of the dust within the AGN torus (Levenson et al. 2007), the dust mineralogy (Sirocky et al. 2008), the grain structure (Fig. 4), and is rather insensitive to the source spectrum. We note that, similarly to the discussion of the extinction curve (Sect. 2.7), the effective optical depth derived from the $10 \mu \mathrm{m}$ silicate absorption feature does not provide a measure, and at best is only a lower limit, of the optical depth of the dust.

Histograms of the strength of the $10 \mu \mathrm{m}$ silicate band are shown in Fig. 8 for the AGN library as a whole and disk-only models. The latter are selected from the library of models without clumps $(\eta=0)$.

For Seyfert 1s the observed feature strength ranges for emission bands between $-0.15 \leq \tau_{\mathrm{Si}} \leq-0.01$ and for absorption bands between $0.06 \leq \tau_{\mathrm{Si}} \leq 1.5$, for quasars between $-0.3 \leq \tau_{\mathrm{Si}} \leq-0.01$ and $\tau_{\mathrm{Si}} \leq 0.62$, respectively (Thompson et al. 2009). Schartmann et al. (2008) observe a strength of the absorption band for type 2 AGNs between $0.2 \leq \tau_{\mathrm{Si}} \leq 3.5$.
The AGN library accounts well for the observed scatter (Fig. 8). In the setup of disk-only models absorption features stronger than $\tau_{\mathrm{Si}} \geq 0.8$ are not present. Levenson et al. (2007) report a mean feature strength for Seyfert 1 s around $\bar{\tau}_{\mathrm{Si}} \sim-0.2$ and for Seyfert $2 \mathrm{~s}$ around $\bar{\tau}_{\mathrm{Si}} \sim 0.2$. Average values derived from the distributions functions presented in Fig. 8 are about the same. We cannot identify a striking need to postulate clumpy AGN models when explaining observed feature strengths of the $10 \mu \mathrm{m}$ silicate band. Arbitrary clump distributions ease accounting for silicate features observed at any strength and viewing angle of the AGNs.

Disk configurations where the $10 \mu \mathrm{m}$ band is in absorption or emission can be seen in the distribution functions presented in Fig. 7. The distribution of the central wavelength position $\lambda_{c}$ of the silicate band is shown in the top panels of Fig. 7. In the silicate band the cross section $K_{\mathrm{Si}}$ peaks at $9.5 \mu \mathrm{m}$ and the exact position depends on the choice of optical constants. Absorption features detected in the SED match this position with some scatter that is explained by radiative transfer effects. Noticeably for most models $\lambda_{\mathrm{c}}$ of the emission feature is shifted to $10.5-11.9 \mu \mathrm{m}$. The shift of the extrema to such long wavelengths is naturally explained assuming that the emission feature is optically thin, so that the observed flux is estimated by 


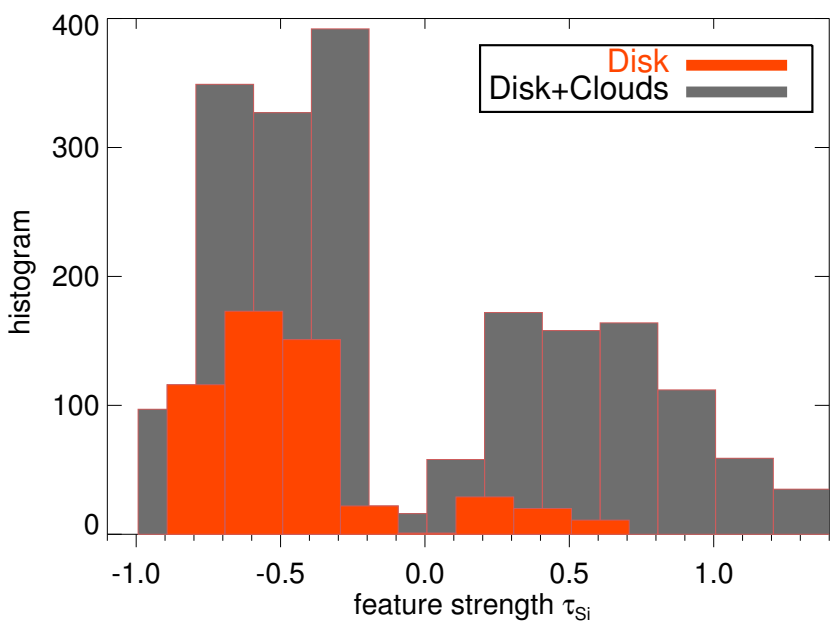

Fig. 8. Distribution of the strength of the $10 \mu \mathrm{m}$ silicate band (Eq. (7)) for the AGN library (labeled "Disk+Clouds", gray) and for the diskonly models (red). Emission features have $\tau_{\mathrm{Si}}<0$ and absorption features $\tau_{\mathrm{Si}}>0$. The low frequency tail of the distribution function is truncated at $\tau_{\mathrm{Si}}=1.4$.

$F \sim K \times B\left(T_{\mathrm{d}}\right)$. The folding of $K$ with the steeply rising Planck function $B\left(T_{\mathrm{d}}\right)$ of the dust at temperature $T_{\mathrm{d}}$ results in the observed wavelength shift (Siebenmorgen et al. 2005; Nikutta et al. 2009).

The silicate feature may appear in emission whenever there is an optically thin view to the inner, hot regions of the disk. This may happen for any $\tau_{V \text {,mid }}$ of the disk midplane, inner radii and viewing directions (Fig. 7, right). Most of the emission bands in disk-only models are seen close to face-on views. There are only a few disk-only models that have sufficient dust extinction so that the silicate band is in absorption. These are models with $r_{\text {in }} \geq \mathrm{pc} / 4, \tau_{V \text {,mid }} \gtrsim 100$, and the disk view needs to be $\theta \gtrsim 60^{\circ}$. When clumps are considered (Fig. 7, left) the silicate band may appear either in absorption or emission for an almost arbitrary choice of disk configurations and viewing angles. It is striking that there are clumpy models displaying the emission band in edge-on view. There is a rather constant decrease in the number of "Disk+Clouds" models that show the emission band when the inner radius or the optical depth is increased, and for the absorption band the opposite trend is seen. All this is consistent and explained by the corresponding increase or decrease in the dust extinction and temperature.

\subsection{Apparent versus intrinsic AGN luminosity}

Because of the assumed spectral shape of the AGN (Eq. (2)), photons are predominantly emitted from the central source at far-UV to near-IR wavelengths. Dust near the AGN scatters and absorbs these photons and re-emits this radiation in the IR. By integrating over the observed fluxes one derives the "apparent" AGN luminosity $L_{\mathrm{obs}}$. One is however interested in deriving from observations the intrinsic luminosity of the AGN source $L_{\mathrm{AGN}}$. The AGN library provides a utility that allows the conversion of the apparent (observed) luminosity to the intrinsic luminosity of the AGN.

In the models the apparent luminosity is computed from photons emitted into a given viewing bin. We compute the observed luminosity for models where dust is considered or neglected. For the latter, the no-dust models, the luminosity is constant because the central source emits isotropically. Therefore in one of the nine viewing bins used, the luminosity $L_{11} / 9=4 \pi D^{2} F_{\text {nd }}(\theta)$, where in the library $D=50 \mathrm{Mpc}$, and $F_{\text {nd }}(\theta)$ is the flux integrated over frequency in a particular $\theta$ direction for the "no dust" model. For models with dust the luminosity is computed from the flux as given in the library, and $L_{\text {obs }}(\theta)=4 \pi D^{2} F(\theta)$. We define the anisotropy conversion factor

$\epsilon=\frac{F(\theta)}{F_{\mathrm{nd}}}$.

The conversion factor becomes larger than 1 whenever there are more photons detected in a viewing bin than originally emitted in that direction from the central source. The conversion factor $\epsilon$ allows one to derive the intrinsic AGN luminosity $L_{\mathrm{AGN}}$. The ratio of the observed-to-intrinsic luminosity is given by

$L_{\mathrm{AGN}}=9 \frac{L_{\mathrm{obs}}(\theta)}{\epsilon}$.

The distribution functions of $L_{\mathrm{obs}} / L_{\mathrm{AGN}}$ are shown in Fig. 9 for different AGN models. Typically the apparent luminosity is about two times larger for type Is (face-on) than for type IIs (edge-on). The fact that for the same intrinsic AGN luminosity, models which correspond to type 1 sources emit more IR radiation than type $2 \mathrm{~s}$ is clearly visible in the SEDs (Fig. 5). Such an increase in the IR radiation is observed in a sample of 3CR sources (Siebenmorgen et al. 2004a). For face-on views there are often more photons radiated into the beam than originally emitted from the source, hence $L_{\mathrm{obs}} / L_{\mathrm{AGN}}>1$. Models with clumps show for edge-on views a broader distribution in $L_{\mathrm{obs}} / L_{\mathrm{AGN}}$ than models without clumps. The impact of the cloud filling factor $\eta$ is shown for models without a disk in Fig. 9 (bottom). For viewing directions with a single cloud along the line of sight typically $40 \%$ of the AGN luminosity is observed. This may happen for AGNs without a disk and with a small number of clouds ( $\eta=1.5 \%$ ). When there are more clouds $L_{\mathrm{obs}} / L_{\mathrm{AGN}}$ increases and ranges between $0.7-1.3$.

We note that the apparent AGN luminosity, which is usually what is quoted in the literature, must simply be divided by $\epsilon$ in order to estimate the intrinsic luminosity of the AGN Eq. (9). The conversion factor $\epsilon$ is the inverse of the anisotropy factor $A$ defined by Efstathiou (2006).

\section{Testing the SED library}

In this section we fit the observed SEDs of a number of representative objects from the near-IR to the submillimeter including Spitzer/IRS spectroscopy, Herschel photometry of distant high luminosity AGNs and high spatial resolution near- and mid-IR observations of nearby, low luminosity Seyfert nuclei. Furthermore, we test that sources of the library would be classified as AGN when applying the empirical mid-IR selection criteria by Stern et al. (2005). First, we test how much of the observed SED can be explained by pure AGN activity (Fritz et al. 2006). As a second step, we combine the predicted SED from the AGN library with an additional starburst (SB) component. We call such combination "AGN+SB" models. To this end we select elements from the SED library of starburst models of Siebenmorgen \& Krügel (2007). We consider the starbursts radius $R_{\mathrm{SB}}$, the luminosity $L_{\mathrm{SB}}$, the visual extinction $A_{\mathrm{V}}$, and the ratio of the luminosity that is due to OB stars $\eta_{\mathrm{OB}}$ as free parameter and keep the hot spot density constant $\eta_{\mathrm{HS}}=10^{4} \mathrm{~cm}^{-3}$. In addition, to better match data below $2 \mu \mathrm{m}$ we add an extra component. For quasars we use beamed synchrotron emission, which is approximated by a power-law, and for the galaxies stellar light from the host, which is approximated by a blackbody. The extra 

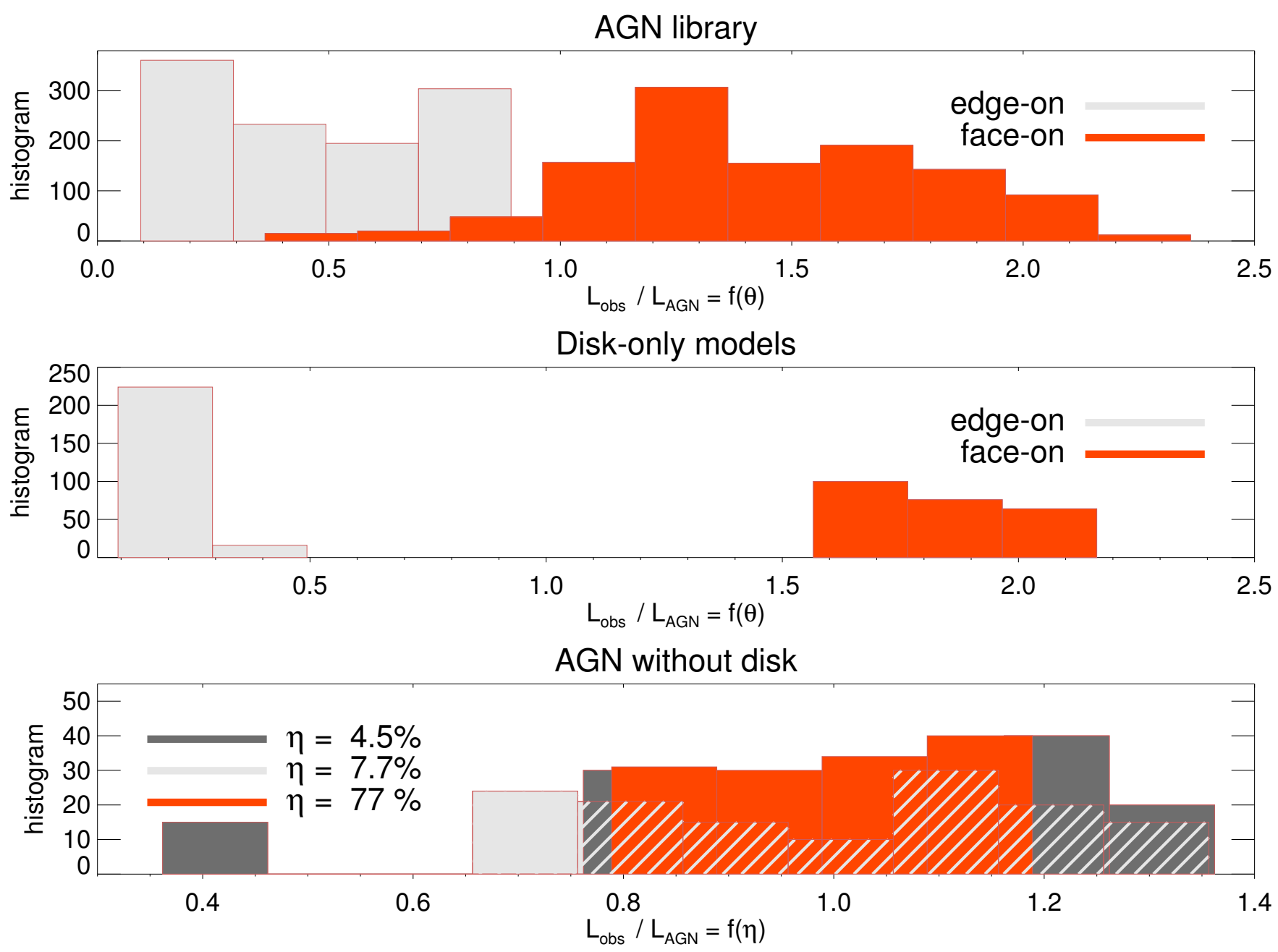

Fig. 9. Histograms of the apparent-to-intrinsic AGN luminosity (Eq. (9)). Results are given for the complete AGN library (top), and for disk-only models (middle) in face-on $\left(\theta \lesssim 43^{\circ}\right)$ and edge-on $\left(\theta \gtrsim 73^{\circ}\right)$ views. The luminosity ratio is shown for the volume filling factor of the clouds $\eta$ (bottom) for models without the homogeneous disk.

components add a minor contribution to the photometry between the thermal IR and the submillimeter. Nevertheless we subtract the flux of the extra components from the data when fitting the dust emission. For the latter fit we use a Bayesian formalism that utilizes the Markov Chain Monte Carlo algorithm by Johnson et al. (2013). This general purpose SED fitting tool employs the Metropolis-Hastings algorithm, e.g. Metropolis et al. (1953). The code is able to take any set of SED templates. In our case we use either the pure AGN library or simultaneously the combination of the AGN+SB libraries. The tool returns the requested best-fitting parameter estimates, and in addition construct the confidence levels from the posterior parameter distribution. We verified the accuracy and robustness of that SED fitter against results obtained with traditional least-squares methods. We confirm Johnson et al. (2013) that the program recovers best-fitting values similar to those from traditional methods.

In the fit procedure the Spitzer spectroscopy data are binned to a spectral resolution that adequately reproduces the shape of the SED including the silicate feature. We display the SED in Figs. 10-12 and the best fit parameters including their uncertainties are summarized for the pure AGN models in Table 3 and for the combination of AGN and starburst activity in Table 4. Apart from the inclination, the fit parameters may cover almost the entire parameter range because of the arbitrary cloud distribution. One finds adequate fits (solutions) of the SED using either a combination of a large number of clouds with small optical depth, or models that have a small number of clouds with high optical depth. Such a change of the cloud distribution requires that the inner radius, the optical depth of the disk or other of the free parameters are modified as well.

\subsection{Observations}

We derive aperture photometry on the final images extracted from the Herschel archive and apply correction factors for the encircled energy fraction and colors as given by Balog et al. (2014) and the User Manuals. The background is subtracted and estimated from the mean flux that is measured in a $2^{\prime \prime}$ wide annulus which is placed 1 " farther out than the source aperture.

PACS and SPIRE photometry of 3C 249.1 and 3C 351 (PI: Luis Ho) is available in 6 Herschel filters and summarized in Table 2. The total flux is measured and uncertainties are computed by adding on the statistical error a calibration uncertainty of $7 \%$ for PACS and $5.5 \%$ for SPIRE bands as well as a confusion noise of $5.8,6.3$, and $6.8 \mathrm{mJy}$ in the bands at 250,350 , and $500 \mu \mathrm{m}$, respectively.

PACS photometry of the Seyfert nuclei NGC 1365 (PI: M. Sanchez), NGC 3081 (PI: M. Sanchez), and NGC 4151 (PI: A. Alonsoh) is measured by adjusting a radius of the source aperture that matches the diffraction limit of the Herschel telescope. 


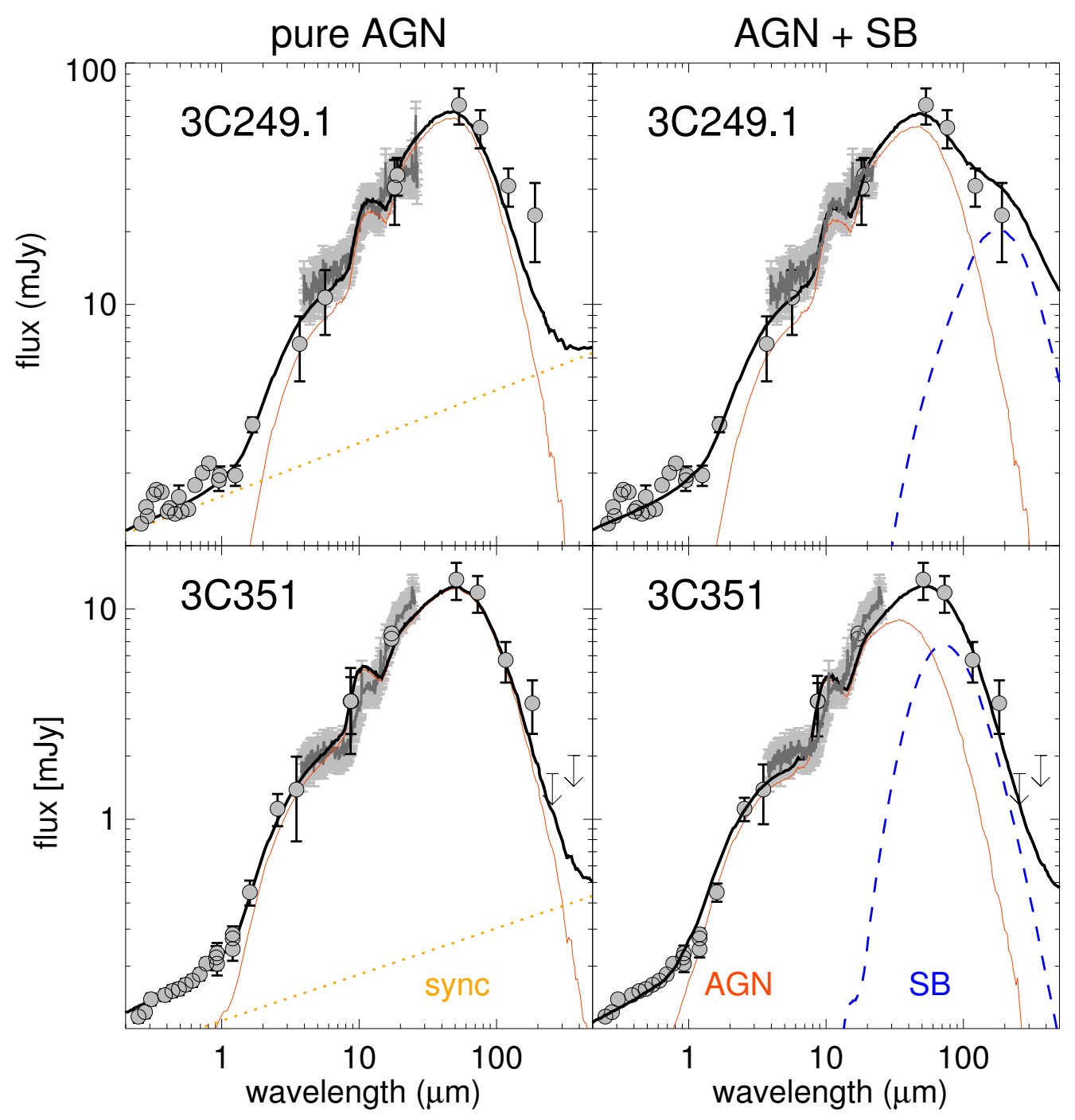

Fig. 10. Rest frame SED of the quasars 3C 249.1 (top) and 3C351 (bottom). Data are from the NASA/IPAC Extragalactic Database (NED), Spitzer/IRS (Leipski et al. 2010) and Herschel (Table 2). A fit using a pure AGN is shown on the left and for a combination (black line) of AGN (red line) and starburst (blue dashed) activity on the right. A synchrotron component (yellow dots) is added to better match the near-IR. Model parameters are given in Table 3.

We apply source radii of $6^{\prime \prime}, 7^{\prime \prime}$, and $12^{\prime \prime}$ at 70,100 , and $160 \mu \mathrm{m}$, respectively (Balog et al. 2014). We center this aperture on the location of the AGN. These galaxies are resolved by Herschel but their AGN tori are not. For the other objects Herschel photometry is obtained from Efstathiou et al. (2013) and Podigachoski et al. (2015).

\subsection{Fits to the quasars $3 C 351$ and $3 C 249.1$}

The near-IR photometry of 3C 351 is fit assuming a contribution of a beamed synchrotron component that we approximate as a power-law $\left(F_{v} \propto v^{\alpha}\right)$ with a slope $\alpha \sim-0.2$. We show that pure AGN models with parameters are summarized in Table 3 account for the complete SED without the need of postulating additional starburst activity.

The best fit of the AGN+SB models shows that the SED can be explained mainly by a torus and a small (8\%, Table 4$)$ contribution from a starburst. The predicted viewing direction is close to face on $\left(\theta \sim 33^{\circ}\right)$. The models reproduce the silicate emission feature of this quasar and the near-IR bump without the need for an additional hot dust component as suggested by
Mor et al. (2009). This is a direct consequence of additional material in the innermost region of the torus that is provided by the homogeneous disk and by clouds in the ionization cones of the AGN. We note that our conclusion that the starburst makes a negligible contribution to the bolometric luminosity is a consequence of the large ratio of $r_{\text {out }} / r_{\text {in }} \sim 170$, and the assumed fluffy grain model. The far-IR emissivity of fluffy grains is much larger than that of ISM dust (Sect. 2.6, Fig. 4).

For 3C 249.1 we find that pure AGN models provide a reasonable fit to the SED and its silicate emission feature without the need for SB activity. There are of course AGN+SB models, similar to $3 \mathrm{C} 351$, that fit the data. Again, we assume synchrotron emission that makes a significant contribution to the near-IR. We find that in this quasar the contribution to the total luminosity from a starburst is $3 \%$ (Table 3 ).

\subsection{Fits to the radio galaxies $3 C 324$ and $3 C 356$}

The first thing that is striking about the two radio galaxies we studied here (3C 324 and 3C 356) is that their SEDs are distinctly different from those of the quasars. They show a much weaker 


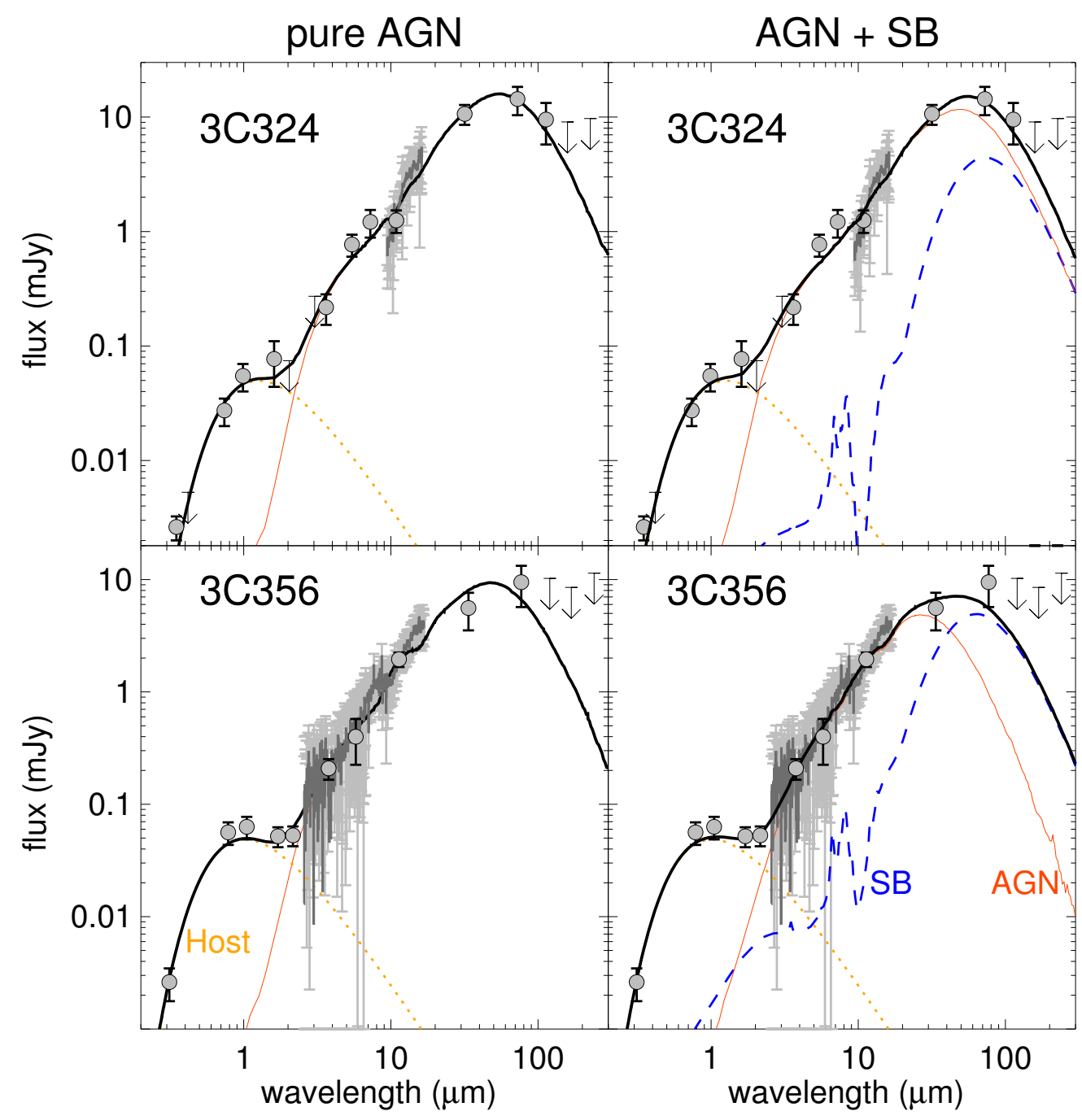

Fig. 11. Rest frame SED of the radio galaxies 3C324 (top) and 3C 356 (bottom). Data are from NED, Spitzer/IRS (Leipski et al. 2010) and Herschel (Podigachoski et al. 2015). A fit using a pure AGN is shown on the left and for a combination (black line) of AGN (red line) and starburst (blue dashed) activity on the right. Stellar light from the host (yellow dots) is added to better match the near-IR. Model parameters are given in Table 3.

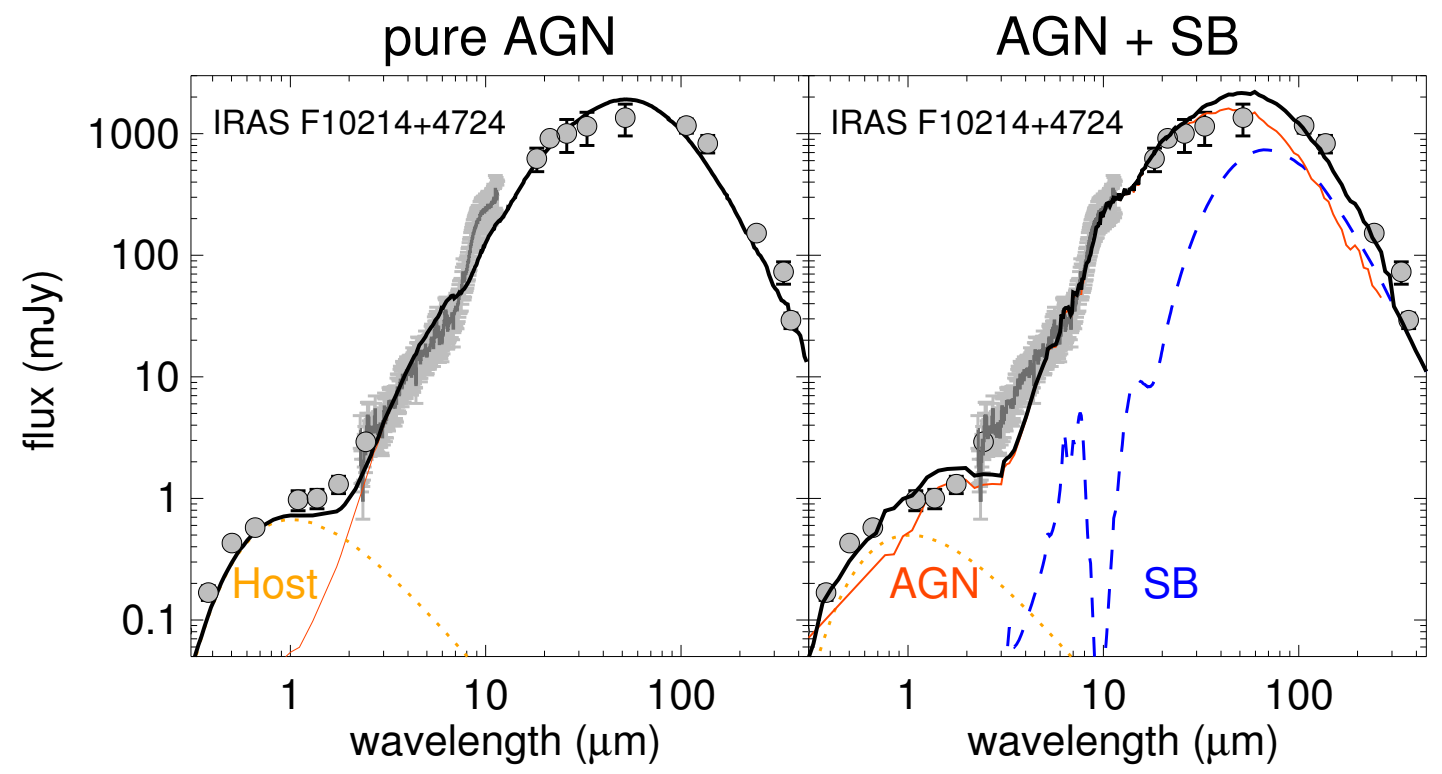

Fig. 12. As Fig. 11 for the hyperluminous IR galaxy IRAS 10214+4724 with data from NED, Spitzer IRS (Hernán-Caballero \& Hatziminaoglou 2011), and MIPS, IRAC and Herschel by Efstathiou et al. (2013). A fit using pure AGN models are shown with (dashed) and without (dotted) clumps. Model parameters are given in Table 3. 
Table 2. Herschel photometry with $1 \sigma$ flux uncertainty.

\begin{tabular}{lrr}
\hline \hline Object & $\begin{array}{r}\text { Filter } \\
(\mu \mathrm{m})\end{array}$ & $\begin{array}{r}\text { Flux } \\
(\mathrm{Jy})\end{array}$ \\
\hline NGC 1365 & 70 & $128 \pm 14$ \\
& 100 & $148 \pm 16$ \\
& 160 & $179 \pm 20$ \\
\hline NGC 3081 & 70 & $2.8 \pm 0.3$ \\
& 100 & $3.1 \pm 0.4$ \\
& 160 & $3.5 \pm 0.4$ \\
\hline NGC 4151 & 70 & $5.2 \pm 0.6$ \\
& 100 & $3.4 \pm 0.4$ \\
& 160 & $4.9 \pm 0.6$ \\
\hline & & \\
& $(\mu \mathrm{m})$ & $(\mathrm{mJy})$ \\
\hline 3C 249.1 & 70 & $87.9 \pm 6.2$ \\
& 100 & $70.9 \pm 5.7$ \\
& 160 & $40.6 \pm 3.3$ \\
& 250 & $30.7 \pm 8.0$ \\
& 350 & $41.7 \pm 8.7$ \\
& 500 & $50.7 \pm 9.4$ \\
\hline 3C 351 & 70 & $189.6 \pm 19.1$ \\
& 100 & $164.4 \pm 16.2$ \\
& 160 & $78.5 \pm 9.3$ \\
& 250 & $48.9 \pm 9.1$ \\
& 350 & $19.6 \pm 7.5$ \\
& 500 & $16.5 \pm 9.2$ \\
\hline
\end{tabular}

near- to mid-IR continuum relative to the far-IR, a completely different slope in the $1-10 \mu \mathrm{m}$ range and evidence of shallow absorption features at $10 \mu \mathrm{m}$. All of these differences are understood in terms of the optically thick torus model and are strongly supportive of the idea of unification. For the radio galaxies we add to the pure AGN torus or the AGN+SB models contributions from black bodies at temperature of 4000-5000 K, which model the emission from old stars in the host galaxies in these objects. The SED of both galaxies are fit by pure AGN models with parameters as given in Table 3 . In the combined models the starburst component is $8 \%$ for $3 \mathrm{C} 324$ and $19 \%$ for $3 \mathrm{C} 356$ of the intrinsic AGN luminosity (Table 4).

\subsection{Fit to the hyperluminous IR galaxy IRAS F10214+4724}

We discuss a fit of the SED of the hyperluminous IR galaxy IRAS F10214+4724 which has an apparent luminosity that exceeds $10^{14} L_{\odot}$ (Rowan-Robinson et al. 1991). It is generally accepted that the enormous luminosity of this galaxy at redshift $z=2.224$ is due to gravitational lensing by a foreground galaxy with magnification between 50 and 100 (Broadhurst \& Lehar 1995; Serjeant et al. 1995) or 15-20 (Deane et al. 2013). Following the detection of a silicate emission feature (Teplitz et al. 2006) in this narrow-lined object, Efstathiou (2006) and Efstathiou et al. (2013) proposed that the emission feature is due to emission from narrow-line region clouds at a temperature of $210 \mathrm{~K}$.

Here we find that the SED of IRAS F10214+4724 can also be fit by a pure AGN model without clouds when a small inner radius or in a "Disk+Clouds" configuration when larger inner radii are assumed (Table 3 ). In the $\mathrm{AGN}+\mathrm{SB}$ models we find the ratio of starburst to AGN luminosity is $20 \%$ (Table 4). The model presented here for IRAS F10214+4724 is similar to that presented in Efstathiou et al. (2013) as in both models the mid-IR emission is predicted to come from the conical region that is associated with the narrow-line region. The dominance of the mid-IR emission of this conical region over the emission from the main body of the disk when the system is viewed approximately edge-on is illustrated in the image (top middle) plotted in Fig. 3. Xie et al. (2014) reported three other local ULIRGS with Spitzer spectroscopy which show similar SEDs to IRAS F10214+4724.

\subsection{Fits to Seyfert nuclei}

García-Burillo et al. (2014) presented ALMA continuum observations of NGC 1068 and find that $r_{\text {out }} / r_{\text {in }} \sim 100$. This is much larger than the value inferred by Alonso-Herrero et al. (2011) $\left(r_{\text {out }} / r_{\text {in }} \sim 6\right)$ who used mid-IR data. Ichikawa et al. (2015) also on the basis of near- and mid-IR data, find that this ratio is less than 20 for a sample Seyferts. It is expected and confirmed by our models that the ratio derived from submillimeter data, which is probing dust at $\sim 30 \mathrm{~K}$ or below, will be larger than the ratio derived from mid-IR data which is probing dust at $300 \mathrm{~K}$.

Lira et al. (2013) find that in 50\% of the Seyferts that are not well fit by the clumpy torus model of Nenkova et al. (2010), a near-IR excess is observed. In our models such a near-IR emission is provided by the homogeneous disk component.

Ramos Almeida et al. (2014b) present high spatial resolution $1-18 \mu \mathrm{m}$ data of a sample of nearby undisturbed Seyfert galaxies with low to moderate amounts of foreground extinction ( $A_{V} \lesssim 5 \mathrm{mag}$ ). We model four objects from their sample (the Seyfert 1s NGC 1365, NGC 4151 and the Seyfert 2s NGC 3081 and NGC 5643) to test the ability of our SED library to fit available nuclear near-IR and mid-IR photometry and spectroscopy. We take high spatial resolution mid-IR spectroscopic observations of NGC 1365 from Burtscher et al. (2013), NGC 3081 and NGC 4151 from Ramos Almeida et al. (2014b), and NGC 5643 from Hönig et al. (2010). Our results are shown in Fig. 13 and the fit parameters are summarized in Table 3. The torus models clearly under-predict the far IR data (plotted as upper limits in the figure) as the emission at these wavelengths is dominated by the host galaxy.

\subsection{Two-color IRAC diagram}

Mid-IR photometry provides a simple technique for identifying active galaxies. Stern et al. (2005) report on Spitzer mid-IR colors, derived from IRAC (Fazio et al. 2004), of nearly 10000 spectroscopically identified AGNs and galaxies. They find that simple mid-IR color criteria provide remarkably robust separation of AGNs from normal galaxies and stars. We indicate the empirical relation in Fig. 14. In that diagram AGNs populate the area in between the dashed lines at [3.6]-[4.5] $\gtrsim 0.3$, whereas galaxies and stars are below at [3.6]-[4.5] $\lesssim 0.3$ and $-0.3<[5.8]-[8.0]<3.2$.

We compute the IRAC colors for each spectrum of the AGN library. Further radiation components that may contribute to the IRAC fluxes are ignored. The IRAC magnitudes of the library are computed using filter curves and flux conversion as described in the IRAC Data Handbook. We find that most sources of the AGN library would be classified as AGN when the empirical mid-IR selection criteria by Stern et al. (2005) is applied. Although there are some additional sources in the area near [5.8]-[8.0] $\gtrsim 2$, these are edge-on sources with high optical depth.

\section{Conclusion}

We assume that dust in the AGN torus is distributed in a clumpy medium or in a homogeneous disk or as a combination of 
R. Siebenmorgen et al.: Self-consistent two-phase AGN torus models: SED library for observers

Table 3. Fitting parameters of pure AGN models for representative objects.

\begin{tabular}{|c|c|c|c|c|c|c|c|c|c|}
\hline & & \multicolumn{5}{|c|}{ Model parameter } & \multicolumn{3}{|c|}{ Derived quantities } \\
\hline Object & Type & $\begin{array}{r}\text { Radius } \\
r_{\text {in }} \\
\left(10^{17} \mathrm{~cm}\right)\end{array}$ & $\begin{array}{l}\text { Filling } \\
\text { factor } \eta\end{array}$ & $\begin{array}{c}\text { Cloud } \\
\tau_{V, \mathrm{cl}}\end{array}$ & $\begin{array}{r}\text { Disk } \\
\tau_{V \text {,mid }}\end{array}$ & $\begin{array}{c}\text { Inc. } \\
\theta \\
\left(^{\circ}\right) \\
\end{array}$ & $\begin{array}{c}\text { Conv. }^{a} \\
\epsilon\end{array}$ & $\begin{array}{c}\text { Radius } \\
R_{\text {in }}^{b} \\
(\mathrm{pc}) \\
\end{array}$ & $\begin{array}{c}\text { Intrinsic AGN } \\
\text { luminosity } L_{\mathrm{AGN}} \\
\log \left(L_{\odot}\right)\end{array}$ \\
\hline NGC 1365 & I & $7.8_{-5}^{+7}$ & $8_{-7}^{+67}$ & $14_{-9}^{+31}$ & $470_{-430}^{+530}$ & $33_{-14}^{+10}$ & $1.34_{-0.02}^{+0.12}$ & $0.1_{-0.06}^{+0.09}$ & $10.23_{-0.04}^{+0.05}$ \\
\hline NGC 4151 & I & $4.8_{-2}^{+4}$ & $3_{-2}^{+75}$ & $40_{-39}^{+5}$ & $230_{-190}^{+770}$ & $43_{-24}^{+9}$ & $2.08_{-0.02}^{+0.12}$ & $0.1_{-0.02}^{+0.41}$ & $10.35_{-0.02}^{+0.02}$ \\
\hline NGC 3081 & II & $3.4_{-3}^{+12}$ & $16_{-14}^{+62}$ & $45_{-44}^{+1}$ & $7_{-6}^{+990}$ & $80_{-27}^{+6}$ & $0.72_{-0.02}^{+0.09}$ & $0.1_{-0.02}^{+0.41}$ & $11.04_{-0.05}^{+0.05}$ \\
\hline NGC 5643 & II & $15_{-12}^{+0.5}$ & $48_{-44}^{+23}$ & $14_{-10}^{+31}$ & $400_{-400}^{+600}$ & $67_{-7}^{+19}$ & $0.84_{-0.19}^{+0.02}$ & $0.3_{-0.2}^{+0.12}$ & $10.65_{-0.02}^{+0.11}$ \\
\hline $3 \mathrm{C} 249.1$ & I & $7.7_{-2}^{+5}$ & $2_{-1}^{+33}$ & $1_{-1}^{+4}$ & $340_{-240}^{+560}$ & $60_{-7}^{+7}$ & $0.82_{-0.26}^{+0.23}$ & $4.8_{-1.3}^{+3.1}$ & $13.57_{-0.17}^{+0.11}$ \\
\hline $3 \mathrm{C} 351$ & I & $7.8_{-4.5}^{+2.2}$ & $14_{-13}^{+60}$ & $1_{-1}^{+4}$ & $840_{-530}^{+160}$ & $43_{-24}^{+17}$ & $2_{-0.05}^{+0.04}$ & $4.7_{-2.7}^{+1.3}$ & $13.54_{-0.02}^{+0.02}$ \\
\hline $3 \mathrm{C} 324$ & II & $15_{-12}^{+0.4}$ & $2_{-1}^{+74}$ & $40_{-40}^{+5}$ & $290_{-170}^{+700}$ & $67_{-7}^{+6}$ & $0.6_{-0.14}^{+0.12}$ & $20.3_{-16.3}^{+0.6}$ & $14.24_{-0.08}^{+0.12}$ \\
\hline $3 \mathrm{C} 356$ & II & $10_{-7}^{+5}$ & $7_{-5}^{+70}$ & $4_{-4}^{+40}$ & $260_{-260}^{+740}$ & $67_{-7}^{+21}$ & $0.66_{-0.18}^{+0.16}$ & $9.2_{-6.4}^{+4.6}$ & $13.9_{-0.14}^{+0.09}$ \\
\hline $\begin{array}{l}\mathrm{F} 10214^{c} \\
+4724\end{array}$ & $\mathrm{HL}$ & $15_{-5}^{+0.4}$ & $40_{-33}^{+37}$ & $6_{-6}^{+2}$ & $910_{-600}^{+90}$ & $67_{-7}^{+5}$ & $0.66_{-0.15}^{+0.14}$ & - & - \\
\hline
\end{tabular}

Notes. ${ }^{(a)}$ Anisotropic luminosity conversion factor (Eq. (8)). ${ }^{(b)}$ Inner radius of the dust torus $R_{\text {in }}=r_{\text {in }} \sqrt{L_{\mathrm{AGN}} / 10^{11}}$. ${ }^{(c)}$ We do not estimate $R_{\text {in }}$ and $L_{\mathrm{AGN}}$ (Eq. (9)) for the lensed galaxy.

Table 4. Fitting parameters of a combination of AGN and starburst models for representative objects.

\begin{tabular}{|c|c|c|c|c|c|c|c|c|c|c|c|c|c|}
\hline \multirow[b]{2}{*}{ Object } & \multicolumn{5}{|c|}{ AGN parameter } & \multicolumn{4}{|c|}{ Starburst parameter } & \multicolumn{4}{|c|}{ Derived Quantities } \\
\hline & $\begin{array}{r}r_{\text {in }} \\
\left(10^{17} \mathrm{~cm}\right) \\
\end{array}$ & $\eta$ & $\tau_{V, \mathrm{cl}}$ & $\tau_{V, \text { mid }}$ & $\begin{array}{c}\theta \theta \\
\left({ }^{\circ}\right) \\
\end{array}$ & $\begin{array}{c}R_{\mathrm{SB}} \\
(\mathrm{kpc})\end{array}$ & $\begin{array}{c}L_{\mathrm{SB}} \\
\log \left(L_{\odot}\right) \\
\end{array}$ & $\begin{array}{c}A_{V} \\
(\mathrm{mag})\end{array}$ & $\begin{array}{l}\eta_{\mathrm{OB}} \\
(\%) \\
\end{array}$ & $\begin{array}{c}\epsilon \\
(\%) \\
\end{array}$ & $\begin{array}{l}\frac{L_{\mathrm{SB}} a}{L_{\mathrm{obs}}} \\
(\%) \\
\end{array}$ & $\begin{array}{c}R_{\text {in }} \\
(\mathrm{pc})\end{array}$ & $\begin{array}{c}L_{\mathrm{AGN}} \\
\log \left(L_{\odot}\right)\end{array}$ \\
\hline 3C 249.1 & $8.7_{-0.9}^{+0.2}$ & $2_{-0.5}^{+21}$ & $2_{-0.3}^{+0.4}$ & $240_{-70}^{+310}$ & $63_{-3}^{+2}$ & $2.0_{-1.3}^{+1.0}$ & $10.75_{-0.1}^{+0.1}$ & $126_{-97}^{+18}$ & $83_{-31}^{+7}$ & $0.82_{-0.01}^{+0.04}$ & $3.4_{-1.5}^{+1.7}$ & $3.9_{-1.3}^{+1.7}$ & $13.28_{-0.01}^{+0.02}$ \\
\hline 3C 351 & $6.4_{-1.2}^{+0.05}$ & $12_{-10}^{+11}$ & $2_{-0.4}^{+0.4}$ & $210_{-37}^{+790}$ & $64_{-30}^{+2}$ & $1.0_{-0.3}^{+1.0}$ & $11.69_{-0.1}^{+0.1}$ & $83_{-29}^{+61}$ & $52_{-12}^{+8}$ & $1.51_{-0.05}^{+0.04}$ & $5.5_{-2.4}^{+2.2}$ & $4.9_{-1.1}^{+2.1}$ & $13.75_{-0.01}^{+0.01}$ \\
\hline $3 \mathrm{C} 324$ & $12.1_{-4.4}^{+0.6}$ & $2_{-1}^{+2}$ & $36_{-16.8}^{+9.4}$ & $314_{-141}^{+233}$ & $68_{-3}^{+4}$ & $1.7_{-1.4}^{+1.3}$ & $11.88_{-0.9}^{+0.3}$ & $67_{-13}^{+77}$ & $46_{-6}^{+44}$ & $0.62_{-0.19}^{+0.02}$ & $13.8_{-6.3}^{+3.3}$ & $9.5_{-0.5}^{+4.4}$ & $13.76_{-0.16}^{+0.01}$ \\
\hline 3C 356 & $3.2_{-0.2}^{+0.9}$ & $1_{-0.7}^{+0.8}$ & $2_{-1.9}^{+2.2}$ & $84_{-18}^{+464}$ & $70_{-4}^{+7}$ & $1.5_{-1.1}^{+1.5}$ & $11.88_{-1.4}^{+0.1}$ & $37_{-28}^{+107}$ & $50_{-10}^{+40}$ & $0.58_{-0.06}^{+0.09}$ & $33.3_{-17.7}^{+11.5}$ & $1.7_{-0.4}^{+1.2}$ & $13.45_{-0.05}^{+0.06}$ \\
\hline $\begin{array}{l}\text { F10214 } \\
+4724\end{array}$ & $3.6_{-0.6}^{+0.5}$ & $40_{-1.5}^{+18}$ & $36_{-1.5}^{+18.1}$ & $34_{-4}^{+222}$ & $27_{-2}^{+11}$ & $1.7_{-1.0}^{+0.3}$ & $11.88_{-0.1}^{+0.1}$ & $92_{-39}^{+51}$ & $45_{-5}^{+44}$ & $1.26_{-0.03}^{+0.05}$ & $19.1_{-8.5}^{+7.6}$ & - & - \\
\hline
\end{tabular}

Notes. Notation as of Table 3. ${ }^{(a)}$ Ratio of the starburst and the apparent AGN luminosity. We consider for the starburst activity the library by Siebenmorgen \& Krügel (2007). 


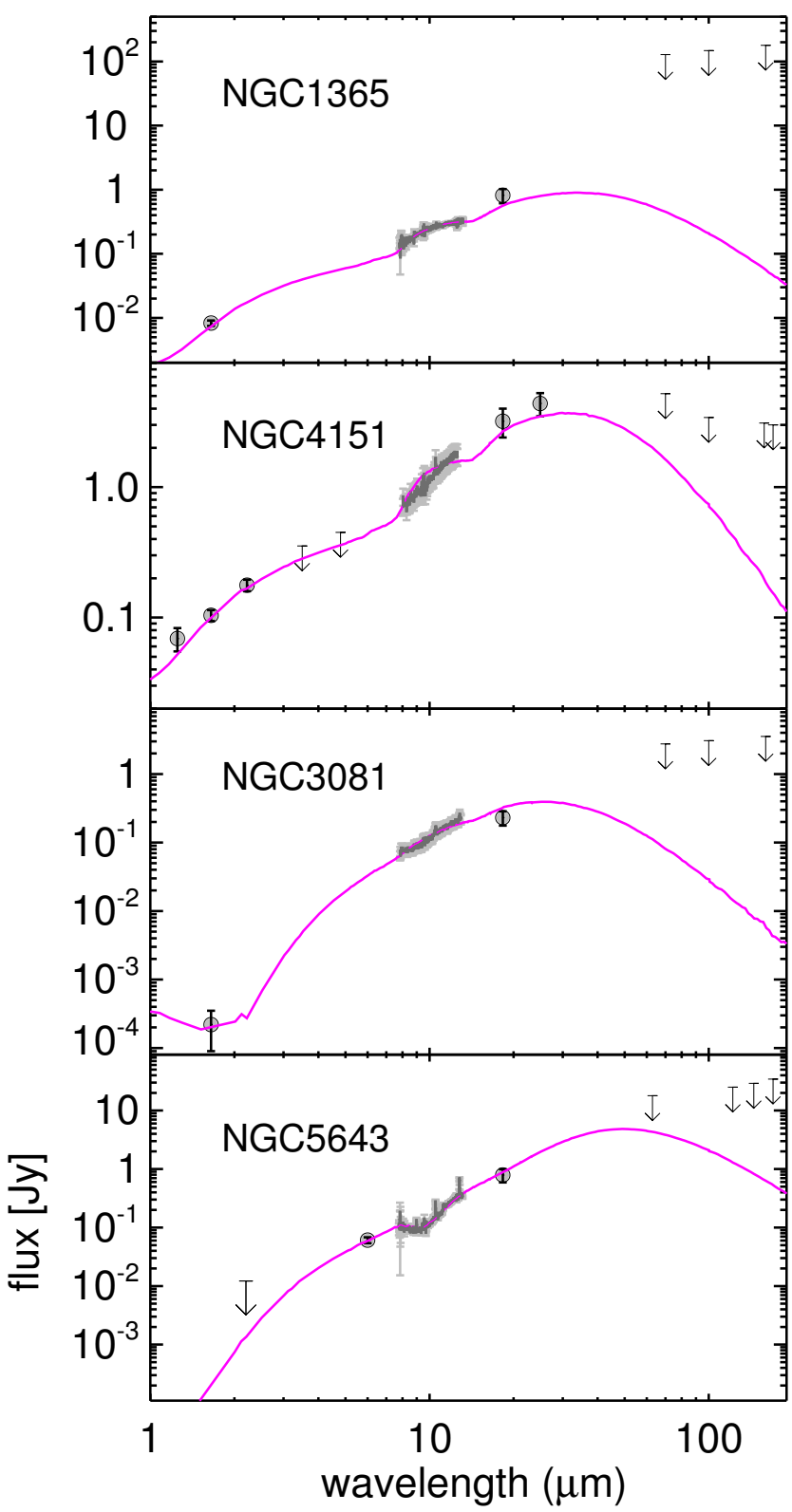

Fig. 13. SED fits using pure AGN models (magenta) of Seyfert nuclei: NGC 1365, NGC 4151, NGC 3081, and NGC 5643. High spatial resolution photometry is from Ramos Almeida et al. (2014b). Ground-based mid-IR spectroscopy of NGC 1365 is performed by Burtscher et al. (2013), NGC 3081 and NGC 4151 by Ramos Almeida et al. (2014b), and NGC 5643 by Hönig et al. (2010), respectively. Far-IR photometry of the nuclear region provided by NED or Herschel (Table 2) are plotted as upper limits of the AGN torus emission. Model parameters are given in Table 3.

the two (i.e. a two-phase medium). We have computed with a self-consistent $3 D$ radiative transfer code the SED of such an AGN structure over a wide range of their basic parameters: the viewing angle, the inner radius, the volume filling factor and optical depth of the clouds, and the optical depth of the disk midplane. We find that details of the cloud properties, such as cloud size, geometrical shape, or their density structure, as well as the presence of clumps such as those detected by X-ray eclipse observations, have a minor impact on the AGN dust emission spectrum.

We visualize the applied 3D dust density structure of the AGN. The AGN torus emits anisotropically and generally

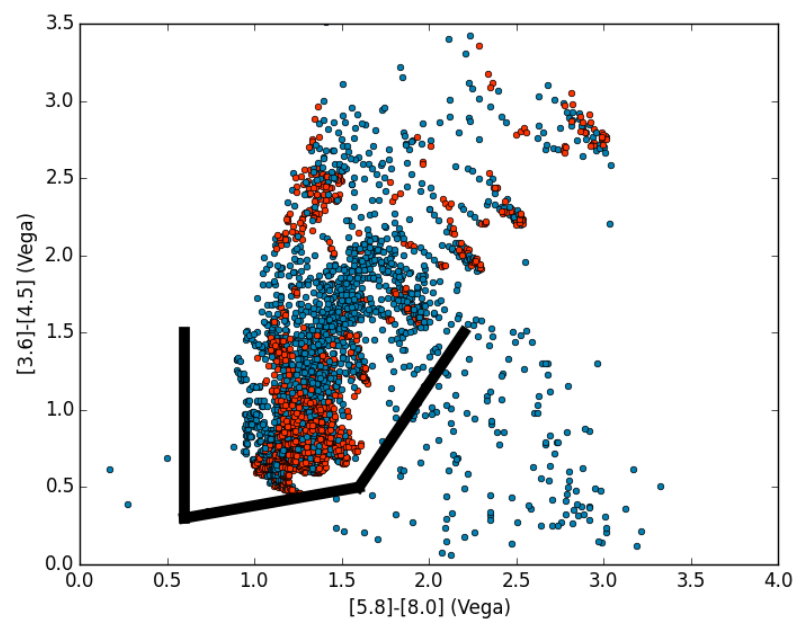

Fig. 14. Spitzer/IRAC mid-IR color diagram. Observed AGNs populate the area in between the black lines at [3.6]-[4.5] $\gtrsim 0.3$ (Stern et al. 2005). Colors derived from the AGN library populate this area. Type I sources with viewing angle $<60^{\circ}$ are shown as red circles and overplotted are type II sources $\left(>60^{\circ}\right)$ as blue circles .

the SED depends on the viewing direction. This happens for wavelengths below the far-IR peak flux. At longer wavelengths the AGN emission becomes isotropic. We also show this in AGN images, where the scattering light $(\sim 1 \mu \mathrm{m})$ and the warm dust emission $(\sim 10 \mu \mathrm{m})$ is anisotropic, while the cold dust emission $(\gtrsim 100 \mu \mathrm{m})$ appears isotropic. The precise wavelength where the AGN becomes isotropic depends on the dust cross section. We find that type I sources emit more IR radiation than type IIs. When the disk material is included there is additional hot dust in the system, which may increase the near-IR flux for face-on views by an order of magnitude.

AGN torus models often consider dust similar to what is assumed for the diffuse ISM. Because of the much higher density and stronger radiation environment in the AGN we favor fluffy grains. Their absorption cross section is generally larger than for ISM dust especially in the far-IR and submillimeter being a factor 10 higher at $1 \mathrm{~mm}$. We show that for unresolved observations, photon scattering within the beam may increase the detected flux and alter the wavelength dependence of the extinction curve. This implies that there is no direct one-to-one link between the observed extinction curve and the wavelength dependence of the dust cross sections. Claims of detecting grain growth in AGN tori that are based on extinction measurements should therefore be treated with caution.

The influence of the model parameters on the SED and their impact on the strength of the $10 \mu \mathrm{m}$ silicate band is discussed. The AGN library accounts well for the observed scatter of the $10 \mu \mathrm{m}$ feature strengths and peak emission. The peak is centered near $10.4 \mu \mathrm{m}$ for ISM dust, whereas it is shifted to even longer wavelengths $(\sim 11.5 \mu \mathrm{m})$ for fluffy grains, and this is more consistent with observations. We cannot identify a striking need to postulate clumpy AGN torus models when explaining observed properties of the silicate band. However, arbitrary clump distributions of the AGN torus ease accounting for silicate features observed at various strengths and viewing angles.

In the UV, optical and near-IR, it may be necessary to add to the library SED a low luminosity component. This may be for type I sources beamed synchrotron radiation or for type II sources stellar light from the galactic disk. These photons escape the torus without interaction. Given a set of data points for a particular AGN, there is a simple procedure to select from 
the library those elements which best match them. If the observations cover the full wavelength bands from the near-IR to submm, one usually finds a library element that fits very well. This is demonstrated for a number of representative type I and type II objects, the famous hyperluminous IR galaxy IRAS F10214+4724, and 4 nearby Seyferts. In all cases, the parameters defining the element are in full accord with what is known about the object from other studies. Interestingly for the luminous AGNs it is possible to explain the SED of these objects with pure AGN models without needing to postulate starburst activity. In a second step we assume a combination of AGN and starburst activity with the goal of estimating an upper limit of the starburst luminosity. We find that the SED of the 5 high luminosity objects studied in this paper, require a starburst luminosity of typically $15 \%$ (with a range between $3-33 \%$ ) of that of the AGN. In our model the starburst contribution to the far-IR emission of the AGN can well be marginal. We also confirm that objects in the library span the range of mid-IR colors as empirically established from observations.

Our library of AGN models promises to be very useful for interpreting the detailed post-Spitzer and post-Herschel SED of large samples of AGNs and (ultra-) luminous IR galaxies in general as well as high spatial resolution imaging observations with large ground-based telescopes and interferometers. The library allows one to constrain the most fundamental properties of the dust torus which is powered by the central AGN. The SED library also provides a utility that allows converting the observed (apparent) AGN luminosity, which is usually what is quoted in the literature, to the intrinsic (true) luminosity of the AGN source.

Acknowledgements. We are grateful to Endrik Krügel for helpful discussions and Bruno Altieri for supporting us in the analysis of the Herschel photometry. We also thank Cristina Ramos Almeida and Sebastian Hönig for providing us their mid-IR spectra in electronic form. We are greatful to Seth Johnson for discussions and help concerning the Bayesian SED fitting technique. This research has made use of the NASA/IPAC Extragalactic Database (NED) which is operated by the Jet Propulsion Laboratory, California Institute of Technology, under contract with the National Aeronautics and Space Administration. We use the NASA/ IPAC Infrared Science Archive, which is operated by the Jet Propulsion Laboratory, California Institute of Technology, under contract with the National Aeronautics and Space Administration.

\section{References}

Alonso-Herrero, A., Ramos Almeida, C., Mason, R., et al. 2011, ApJ, 736, 82 Alonso-Herrero, A., Sánchez-Portal, M., Ramos Almeida, C., et al. 2012, MNRAS, 425, 311

Alonso-Herrero, A., Ramos Almeida, C., Esquej, P., et al. 2014, MNRAS, 443, 2766

Antonucci, R. 1993, ARA\&A, 31, 473

Asmus, D., Hönig, S. F., Gandhi, P., Smette, A., \& Duschl, W. J. 2014, MNRAS, 439, 1648

Balog, Z., Müller, T., Nielbock, M., et al. 2014, Exp. Astron., 37, 129

Bjorkman, J. E., \& Wood, K. 2001, ApJ, 554, 615

Braatz, J. A., Wilson, A. S., Gezari, D. Y., Varosi, F., \& Beichman, C. A. 1993, ApJ, 409, L5

Broadhurst, T., \& Lehar, J. 1995, ApJ, 450, L41

Burtscher, L., Meisenheimer, K., Tristram, K. R. W., et al. 2013, A\&A, 558, A149

Cameron, M., Storey, J. W. V., Rotaciuc, V., et al. 1993, ApJ, 419, 136

Combes, F., García-Burillo, S., Casasola, V., et al. 2014, A\&A, 565, A97

Deane, R. P., Rawlings, S., Garrett, M. A., et al. 2013, MNRAS, 434, 3322

Draine, B. T. 2003, ApJ, 598, 1017

Dullemond, C. P., van Zadelhoff, G. J., \& Natta, A. 2002, A\&A, 389, 464

Efstathiou, A. 2006, MNRAS, 371, L70

Efstathiou, A., \& Rowan-Robinson, M. 1991, MNRAS, 252, 528

Efstathiou, A., \& Rowan-Robinson, M. 1995, MNRAS, 273, 649

Efstathiou, A., Hough, J. H., \& Young, S. 1995, MNRAS, 277, 1134

Efstathiou, A., Christopher, N., Verma, A., \& Siebenmorgen, R. 2013, MNRAS, 436,1873
Efstathiou, A., Pearson, C., Farrah, D., et al. 2014, MNRAS, 437, L16

Elitzur, M., \& Shlosman, I. 2006, ApJ, 648, L101

Esquej, P., Alonso-Herrero, A., González-Martín, O., et al. 2014, ApJ, 780, 86 Fazio, G. G., Hora, J. L., Allen, L. E., et al. 2004, ApJS, 154, 10

Feltre, A., Hatziminaoglou, E., Fritz, J., \& Franceschini, A. 2012, MNRAS, 426, 120

Fritz, J., Franceschini, A., \& Hatziminaoglou, E. 2006, MNRAS, 366, 767

García-Burillo, S., Combes, F., Usero, A., et al. 2014, A\&A, 567, A125

González-Martín, O., Rodríguez-Espinosa, J. M., Díaz-Santos, T., et al. 2013, A\&A, 553, A35

Granato, G. L., \& Danese, L. 1994, MNRAS, 268, 235

Hao, L., Spoon, H. W. W., Sloan, G. C., et al. 2005, ApJ, 625, L75

Hao, L., Weedman, D. W., Spoon, H. W. W., et al. 2007, ApJ, 655, L77

Hatziminaoglou, E., Hernán-Caballero, A., Feltre, A., \& Piñol Ferrer, N. 2015, ApJ, 803, 110

Henyey, L. G., \& Greenstein, J. L. 1941, ApJ, 93, 70

Hernán-Caballero, A., \& Hatziminaoglou, E. 2011, MNRAS, 414, 500

Heymann, F., \& Siebenmorgen, R. 2012, ApJ, 751, 27

Hofmeister, A. M., Pitman, K. M., Goncharov, A. F., \& Speck, A. K. 2009, ApJ, 696,1502

Hönig, S. F., \& Kishimoto, M. 2010, A\&A, 523, A27

Hönig, S. F., Beckert, T., Ohnaka, K., \& Weigelt, G. 2006, A\&A, 452, 459

Hönig, S. F., Kishimoto, M., Gandhi, P., et al. 2010, A\&A, 515, A23

Hönig, S. F., Kishimoto, M., Antonucci, R., et al. 2012, ApJ, 755, 149

Hönig, S. F., Kishimoto, M., Tristram, K. R. W., et al. 2013, ApJ, 771, 87

Ichikawa, K., Packham, C., Ramos Almeida, C., et al. 2015, ApJ, 803, 57

Jaffe, W., Meisenheimer, K., Röttgering, H. J. A., et al. 2004, Nature, 429, 47

Johnson, S. P., Wilson, G. W., Tang, Y., \& Scott, K. S. 2013, MNRAS, 436, 2535

Kawaguchi, T., \& Mori, M. 2011, ApJ, 737, 105

Kishimoto, M., Hönig, S. F., Beckert, T., \& Weigelt, G. 2007, A\&A, 476, 713

Kishimoto, M., Hönig, S. F., Antonucci, R., et al. 2011, A\&A, 527, A121

Krolik, J. H., \& Begelman, M. C. 1988, ApJ, 329, 702

Krügel, E. 2009, A\&A, 493, 385

Krügel, E., \& Siebenmorgen, R. 1994, A\&A, 288, 929

Lawrence, A., Rowan-Robinson, M., Efstathiou, A., et al. 1991, MNRAS, 248, 91

Leipski, C., Haas, M., Willner, S. P., et al. 2010, ApJ, 717, 766

Levenson, N. A., Sirocky, M. M., Hao, L., et al. 2007, ApJ, 654, L45

Lira, P., Videla, L., Wu, Y., et al. 2013, ApJ, 764, 159

Low, J., \& Kleinmann, D. E. 1968, AJ, 73, 868

Lucy, L. B. 1999, A\&A, 344, 282

Maiolino, R., Marconi, A., Salvati, M., et al. 2001, A\&A, 365, 28

Markowitz, A. G., Krumpe, M., \& Nikutta, R. 2014, MNRAS, 439, 1403

Mason, R. E., Levenson, N. A., Packham, C., et al. 2007, ApJ, 659, 241

Mason, R. E., Lopez-Rodriguez, E., Packham, C., et al. 2012, AJ, 144, 11

Mathis, J. S., \& Whiffen, G. 1989, ApJ, 341, 808

Mathis, J. S., Rumpl, W., \& Nordsieck, K. H. 1977, ApJ, 217, 425

Mendoza-Castrejón, S., Dultzin, D., Krongold, Y., González, J. J., \& Elitzur, M. 2015, MNRAS, 447, 2437

Metropolis, N., Rosenbluth, A. W., Rosenbluth, M. N., Teller, A. H., \& Teller, E. 1953, J. Chem. Phys., 21, 1087

Mor, R., Netzer, H., \& Elitzur, M. 2009, ApJ, 705, 298

Natta, A., \& Panagia, N. 1984, ApJ, 287, 228

Nenkova, M., Ivezić, Ž., \& Elitzur, M. 2002, ApJ, 570, L9

Nenkova, M., Sirocky, M. M., Nikutta, R., Ivezić, Ž., \& Elitzur, M. 2010, ApJ, 723,1827

Netzer, H. 1987, MNRAS, 225, 55

Netzer, H. 2015, ARA\&A, 53, 365

Nikutta, R., Elitzur, M., \& Lacy, M. 2009, ApJ, 707, 1550

Pascucci, I., Wolf, S., Steinacker, J., et al. 2004, A\&A, 417, 793

Pier, E. A., \& Krolik, J. H. 1992, ApJ, 401, 99

Pier, E. A., \& Krolik, J. H. 1993, ApJ, 418, 673

Podigachoski, P., Barthel, P. D., Haas, M., et al. 2015, A\&A, 575, A80

Ramos Almeida, C., Levenson, N. A., Alonso-Herrero, A., et al. 2011, ApJ, 731, 92

Ramos Almeida, C., Alonso-Herrero, A., Esquej, P., et al. 2014a, MNRAS, 445, 1130

Ramos Almeida, C., Alonso-Herrero, A., Levenson, N. A., et al. 2014b, MNRAS, 439, 3847

Rees, M. J., Silk, J. I., Werner, M. W., \& Wickramasinghe, N. C. 1969, Nature, 223, 788

Roche, P. F., Aitken, D. K., Smith, C. H., \& Ward, M. J. 1991, MNRAS, 248, 606

Roche, P. F., Packham, C., Telesco, C. M., et al. 2006, MNRAS, 367, 1689

Rowan-Robinson, M. 1995, MNRAS, 272, 737

Rowan-Robinson, M., Broadhurst, T., Oliver, S. J., et al. 1991, Nature, 351, 719

Rowan-Robinson, M., \& Crawford, J. 1989, MNRAS, 238, 523

Rowan-Robinson, M., \& Efstathiou, A. 1993, MNRAS, 263, 675 
Rowan-Robinson, M., Efstathiou, A., Lawrence, A., et al. 1993, MNRAS, 261, 513

Ruschel-Dutra, D., Pastoriza, M., Riffel, R., Sales, D. A., \& Winge, C. 2014, MNRAS, 438, 3434

Sales, D. A., Pastoriza, M. G., Riffel, R., et al. 2011, ApJ, 738, 109

Schartmann, M., Meisenheimer, K., Camenzind, M., et al. 2008, A\&A, 482, 67

Schartmann, M., Wada, K., Prieto, M. A., Burkert, A., \& Tristram, K. R. W. 2014, MNRAS, 445, 3878

Schweitzer, M., Groves, B., Netzer, H., et al. 2008, ApJ, 679, 101

Scicluna, P., \& Siebenmorgen, R. 2015, A\&A, in press, DOI: $10.1051 / 0004-6361 / 201323149$

Serjeant, S., Lacy, M., Rawlings, S., King, L. J., \& Clements, D. L. 1995, MNRAS, 276, L31

Shakura, N. I., \& Sunyaev, R. A. 1976, MNRAS, 175, 613

Siebenmorgen, R., \& Heymann, F. 2012, A\&A, 539, A20

Siebenmorgen, R., \& Krügel, E. 2007, A\&A, 461, 445

Siebenmorgen, R., Freudling, W., Krügel, E., \& Haas, M. 2004a, A\&A, 421, 129

Siebenmorgen, R., Krügel, E., \& Spoon, H. W. W. 2004b, A\&A, 414, 123

Siebenmorgen, R., Haas, M., Krügel, E., \& Schulz, B. 2005, A\&A, 436, L5

Siebenmorgen, R., Voshchinnikov, N. V., \& Bagnulo, S. 2014, A\&A, 561, A82

Sirocky, M. M., Levenson, N. A., Elitzur, M., Spoon, H. W. W., \& Armus, L. 2008, ApJ, 678, 729
Spoon, H. W. W., Keane, J. V., Tielens, A. G. G. M., et al. 2002, A\&A, 385, 1022

Spoon, H. W. W., Marshall, J. A., Houck, J. R., et al. 2007, ApJ, 654, L49

Stalevski, M., Fritz, J., Baes, M., Nakos, T., \& Popović, L. Č. 2012a, MNRAS, 420, 2756

Stalevski, M., Fritz, J., Baes, M., \& Popovic, L. C. 2012b, in Torus Workshop, eds. R. Mason, A. Alonso-Herrero, \& C. Packham, 170

Stern, D., Eisenhardt, P., Gorjian, V., et al. 2005, ApJ, 631, 163

Sturm, E., Schweitzer, M., Lutz, D., et al. 2005, ApJ, 629, L21

Suganuma, M., Yoshii, Y., Kobayashi, Y., et al. 2006, ApJ, 639, 46

Teplitz, H. I., Armus, L., Soifer, B. T., et al. 2006, ApJ, 638, L1

Thompson, G. D., Levenson, N. A., Uddin, S. A., \& Sirocky, M. M. 2009, ApJ, 697, 182

Tristram, K. R. W., Meisenheimer, K., Jaffe, W., et al. 2007, A\&A, 474, 837

Tristram, K. R. W., Raban, D., Meisenheimer, K., et al. 2009, A\&A, 502, 67

Tristram, K. R. W., Burtscher, L., Jaffe, W., et al. 2014, A\&A, 563, A82

Voit, G. M. 1992, MNRAS, 258, 841

Wolf, S., Fischer, O., \& Pfau, W. 1998, A\&A, 340, 103

Xie, Y., Hao, L., \& Li, A. 2014, ApJ, 794, L19

Xu, C. K., Cao, C., Lu, N., et al. 2014, ApJ, 787, 48

Zubko, V. G., Mennella, V., Colangeli, L., \& Bussoletti, E. 1996, MNRAS, 282, 1321 